\title{
The low lying zeros of a GL(4) and a GL(6) family of $\boldsymbol{L}$-functions
}

\author{
Eduardo Dueñez and Steven J. Miller
}

\begin{abstract}
We investigate the large weight $(k \rightarrow \infty)$ limiting statistics for the low lying zeros of a GL(4) and a GL(6) family of $L$-functions, $\left\{L(s, \phi \times f): f \in H_{k}\right\}$ and $\left\{L\left(s, \phi \times \operatorname{sym}^{2} f\right)\right.$ : $\left.f \in H_{k}\right\}$; here $\phi$ is a fixed even Hecke-Maass cusp form and $H_{k}$ is a Hecke eigenbasis for the space $H_{k}$ of holomorphic cusp forms of weight $k$ for the full modular group. Katz and Sarnak conjecture that the behavior of zeros near the central point should be well modeled by the behavior of eigenvalues near 1 of a classical compact group. By studying the 1and 2-level densities, we find evidence of underlying symplectic and $\mathrm{SO}$ (even) symmetry, respectively. This should be contrasted with previous results of Iwaniec-Luo-Sarnak for the families $\left\{L(s, f): f \in H_{k}\right\}$ and $\left\{L\left(s, \operatorname{sym}^{2} f\right): f \in H_{k}\right\}$, where they find evidence of orthogonal and symplectic symmetry, respectively. The present examples suggest a relation between the symmetry type of a family and that of its twistings, which will be further studied in a subsequent paper. Both the GL(4) and the GL(6) families above have all even functional equations, and neither is naturally split from an orthogonal family. A folklore conjecture states that such families must be symplectic, which is true for the first family but false for the second. Thus, the theory of low lying zeros is more than just a theory of signs of functional equations. An analysis of these families suggest that it is the second moment of the Satake parameters that determines the symmetry group.
\end{abstract}

\section{Contents}

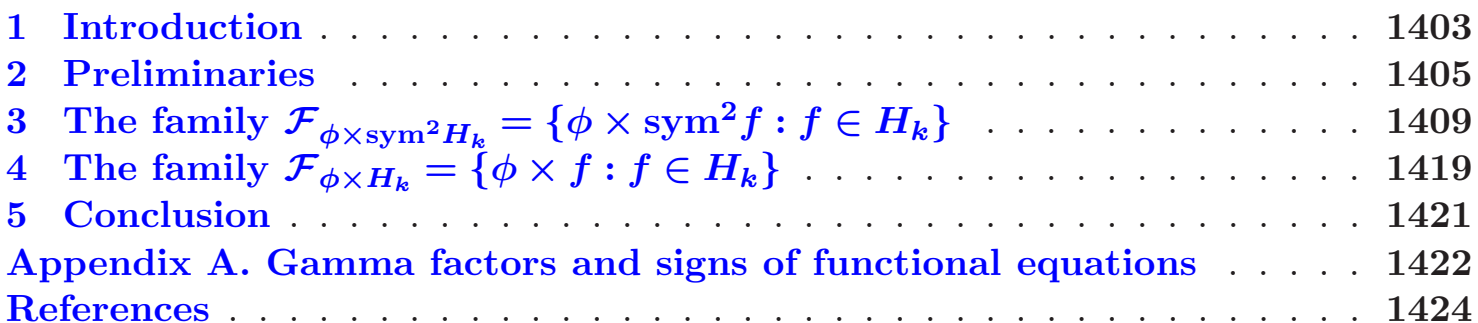

\section{Introduction}

Assuming the generalized Riemann hypothesis (GRH), the non-trivial zeros of any $L$-function lie on its critical line, and therefore it is possible to investigate the statistics of its normalized zeros.

Received 28 June 2005, accepted in final form 6 April 2006.

2000 Mathematics Subject Classification 11M26 (primary), 11M41, 15A52 (secondary).

Keywords: low lying zeros, $n$-level density, random matrix theory, cuspidal newforms, Maass forms.

We thank Wenzhi Luo and Peter Sarnak for suggesting this problem, as well as for many enlightening conversations, and Jim Cogdell for valuable comments in writing the appendix to this article. The first-named author was partly supported by an EPSRC Grant.

This journal is (C) Foundation Compositio Mathematica 2006. 


\section{E. Dueñez And S. J. Miller}

The general philosophy, born out of many examples and proven cases in function fields [CFKRS05, KS99a, KS99b, KS03, ILS00], is that the statistical behavior of eigenvalues of random matrices (respectively, random matrix ensembles) is similar to that of the critical zeros of $L$-functions (respectively, families of $L$-functions).

The global $n$-level correlations of high zeros of primitive automorphic cuspidal $L$-functions, assuming a certain technical restriction, have been found to agree with the corresponding statistics of the eigenvalues of complex hermitian matrices (the Gaussian unitary ensemble, or GUE) [Mon73, Hej94, RS96]. If the technical restriction mentioned above were to be removed, the results on $n$-level correlations would imply that the distributions of the normalized neighbor spacings between consecutive critical zeros of an $L$-function and between GUE eigenvalues coincide, as has been numerically observed [Odl01, Rub98]. The same correlations describe the global statistical behavior of the eigenvalues of other matrix ensembles, most notably of the classical compact groups (orthogonal, unitary, symplectic). As they are insensitive to the effect of finitely many zeros, these correlations miss the behavior of the low-lying zeros, the zeros near the central point $s=\frac{1}{2}$.

Katz and Sarnak [KS99a, KS99b] showed that there is another statistic that can distinguish between the classical compact groups. It is the $n$-level density, and it depends only on eigenvalues near 1. In a number of cases [FI03, Gul05, HM06, HR03, ILS00, Mil04, Roy01, Rub01, You06], the behavior of the low-lying zeros of families of $L$-functions is found to be in agreement with that of the eigenvalues near 1 for random matrices in one of the classical compact groups: unitary, symplectic, and orthogonal (which is further split into $\mathrm{SO}$ (even) and $\mathrm{SO}$ (odd)). This correspondence allows us, at least conjecturally, to assign a definite symmetry type to each family of $L$-functions.

Let $\phi$ be a fixed even Hecke-Maass cusp form and $H_{k}$ a Hecke eigenbasis for the space of holomorphic cusp forms of (even) weight $k$ for the full modular group. Iwaniec, Luo, and Sarnak [ILS00] proved that as $k \rightarrow \infty$, the family $\left\{f: f \in H_{k}\right\}$ has $\mathrm{SO}$ (even) or $\mathrm{SO}$ (odd) symmetry (depending on whether $k / 2$ is even or odd), and the family $\left\{\operatorname{sym}^{2} f: f \in H_{k}\right\}$ has symplectic symmetry. We consider the twisted families $\mathcal{F}_{\phi \times H_{k}}=\left\{\phi \times f: f \in H_{k}\right\}$ and $\mathcal{F}_{\phi \times \operatorname{sym}^{2} H_{k}}=\left\{\phi \times \operatorname{sym}^{2} f:\right.$ $\left.f \in H_{k}\right\}$; the family $\left\{\phi \times \operatorname{sym}^{2} f\right\}$ arose in the work of Luo-Sarnak [LS03], where it is shown to be intimately connected with the relation between the quantum and classical fluctuations of observables on the modular surface. In both families, all functional equations are even. We show that the first family has symplectic symmetry, and the second SO(even). Explicitly, our main results are as follows.

Theorem 1.1. Let $\phi$ be a fixed even Hecke-Maass cusp form. As $k \rightarrow \infty$, for test functions whose Fourier transform has small but computable support, the 1-level density of the family $\mathcal{F}_{\phi \times H_{k}}$ only agrees with symplectic matrices, suggesting that the underlying symmetry of this family is symplectic (and uniquely so).

Theorem 1.2. Let $\phi$ be a fixed even Hecke-Maass cusp form. As $k \rightarrow \infty$, for test functions $g$ with $\operatorname{supp}(\widehat{g}) \subset\left(-\frac{5}{24}, \frac{5}{24}\right)$, the 1-level density of the family $\mathcal{F}_{\phi \times \mathrm{sym}^{2} H_{k}}$ only agrees with $\mathrm{SO}($ even), O and $\mathrm{SO}$ (odd) matrices. For small but computable support, the 2-level density only agrees with $\mathrm{SO}$ (even) matrices, suggesting that the underlying symmetry of this family is $\mathrm{SO}$ (even) (and uniquely so).

For families where the signs of the functional equations are all even and there is no corresponding family with odd functional equations, a 'folklore' conjecture (see, for example, [KS03, p. 2877]) states that the symmetry is symplectic, presumably based on the observation that $\mathrm{SO}$ (even) and $\mathrm{SO}$ (odd) symmetries in the examples known to date arise from splitting orthogonal families according to the sign of the functional equations. A priori, the symmetry type of a family with all functional equations even is either symplectic or $\mathrm{SO}$ (even). All $L$-functions from elements of $\mathcal{F}_{\phi \times H_{k}}$ and $\mathcal{F}_{\phi \times \mathrm{sym}^{2} H_{k}}$ have even functional equations, and neither family seems to naturally arise from splitting sign within a full orthogonal family. By calculating the 1-level density we quickly see the symmetry of the first is symplectic (as predicted); however, the second family has orthogonal symmetry (we cannot 
distinguish between $\mathrm{SO}$ (even), $\mathrm{O}$, and $\mathrm{SO}$ (odd) due to the small-support restriction on the allowable test functions). By calculating the 2-level density for the second family, we can discard $\mathrm{O}$ and $\mathrm{SO}$ (odd). Thus, our calculations are only consistent with the symmetry being SO(even). As our purpose is to show that the theory of low-lying zeros is more than just a theory of signs of functional equations, we do not concern ourselves with obtaining optimal bounds in terms of support, instead simplifying the arguments but still distinguishing the various classical compact group candidates.

In studying the symmetry groups of $\mathcal{F}_{\phi \times H_{k}}$ and $\mathcal{F}_{\phi \times \text { sym }^{2} H_{k}}$, we see that twisting a family with orthogonal (respectively, symplectic) symmetry by a fixed GL(2) form flips the symmetry to symplectic (respectively, orthogonal). The effect on the symmetry group by GL $(n)$ twisting (by a fixed form, or by a second family) in some cases will be described in a subsequent paper [DM06]. The main result is that, for any family $\mathcal{F}$ satisfying certain technical conditions, we can attach a symmetry constant $c_{\mathcal{F}}$, with $c_{\mathcal{F}}=0(1,-1)$ if the family is unitary (symplectic, orthogonal). For such families $\mathcal{F}$ and $\mathcal{G}$, the family $\mathcal{F} \times \mathcal{G}$ (Rankin-Selberg convolution) has symmetry constant $c_{\mathcal{F} \times \mathcal{G}}=c_{\mathcal{F}} \cdot c_{\mathcal{G}}$ (compare with [KS99a]). In other words, the symmetry of a product of families is the product of the family symmetries. This is consistent with earlier results and should be compared, for instance, with Rubinstein's work [Rub01] on twisting the symplectic family of quadratic Dirichlet characters by a fixed $\mathrm{GL}(n)$ form.

We assume the GRH for all $L$-functions encountered. Mostly the GRH is used for interpretation purposes (i.e. if the GRH is true, then the non-trivial zeros lie on the critical line, and we may interpret the $n$-level correlations and densities as spacing statistics between ordered zeros), although in a few places the GRH is assumed to simplify the derivation of needed bounds (although these bounds can be derived unconditionally at the cost of a more careful analysis). In $\S 2$ we review the necessary preliminaries. We concentrate on the more difficult GL(6) family in $\S 3$, and merely sketch the changes needed to handle the GL(4) family in $\S 4$; for completeness the details of the calculation of the gamma factors and signs of the functional equations are given in Appendix A. In $\S 5$ we analyze our results for these two families. The evidence suggests that the theory of low-lying zeros is not just a theory of signs of functional equations, but rather more about the second moment of the Satake parameters. In this regard it is similar to the universality Rudnick and Sarnak [RS96] found for the $n$-level correlations of high zeros of a primitive $L$-function $L(s, \pi)$ ( $\pi$ a cuspidal automorphic representation); their results are a consequence of the universality of the second moments of the Satake parameters $a_{\pi}(p)$.

\section{Preliminaries}

\subsection{1- and 2-level densities}

Let $g$ be an even Schwartz test function on $\mathbb{R}$ whose Fourier transform

$$
\widehat{g}(y)=\int_{-\infty}^{\infty} g(x) e^{-2 \pi i x y} d x
$$

has compact support. Let $\mathcal{F}$ be a finite family, all of whose $L$-functions satisfy the GRH. We define the 1-level density associated to $\mathcal{F}$ by

$$
D_{1, \mathcal{F}}(g)=\frac{1}{|\mathcal{F}|} \sum_{f \in \mathcal{F}} \sum_{j} g\left(\frac{\log c_{f}}{2 \pi} \gamma_{f}^{(j)}\right)
$$

where $\frac{1}{2}+i \gamma_{f}^{(j)}$ runs through the non-trivial zeros of $L(s, f)$. Here $c_{f}$ is the analytic conductor of $f$, and gives the natural scale for the low zeros. Since $g$ is Schwartz, only low-lying zeros (i.e. zeros within a distance of about $1 / \log c_{f}$ of the central point) contribute significantly. Thus, the 1-level density is a local statistic which can potentially help in identifying the symmetry type of the family. 


\section{E. Dueñez And S. J. Miller}

Remark 2.1. For technical convenience, as in [ILS00, Roy01] we will modify (2.2) by weighting each $f$ by a factor $w_{f}$ which varies slowly with $f$. These factors simplify the application of the Petersson formula, and (see [ILS00]) can be removed at the cost of additional book-keeping.

Based, in part, on the function-field analysis where $G(\mathcal{F})$ is the monodromy group associated to the family $\mathcal{F}$, it is conjectured that for each reasonable irreducible family of $L$-functions there is an associated symmetry group $G(\mathcal{F})$ (typically one of the following five subgroups of unitary matrices: unitary U, symplectic USp, orthogonal O, $\mathrm{SO}$ (even), $\mathrm{SO}$ (odd)), and that the distribution of critical zeros near $\frac{1}{2}$ mirrors the distribution of eigenvalues near 1 . The five groups have distinguishable 1-level densities.

To evaluate (2.2), one applies the explicit formula, converting sums over zeros to sums over primes. Unfortunately, these prime sums can often only be evaluated for small support. If one allows test functions with $\operatorname{supp}(\widehat{g}) \subset(-\delta, \delta)$, then for any $\delta>0$ the orthogonal, symplectic, and unitary symmetries can be mutually distinguished via their 1-level density. However, if $\delta \leqslant 1$, then the 1-level densities of the three orthogonal types $\mathrm{O}, \mathrm{SO}$ (even), and $\mathrm{SO}$ (odd) cannot be distinguished from one another.

In order to distinguish between the three orthogonal symmetry types we study the 2-level density of the family, defined as follows. Let $g(x)=g_{1}\left(x_{1}\right) g_{2}\left(x_{2}\right)$, each $\widehat{g_{i}}$ of compact support. Then

$$
D_{2, \mathcal{F}}(g)=\frac{1}{|\mathcal{F}|} \sum_{f \in \mathcal{F}} \sum_{j_{1} \neq \pm j_{2}} g_{1}\left(\frac{\log c_{f}}{2 \pi} \gamma_{f}^{\left(j_{1}\right)}\right) g_{2}\left(\frac{\log c_{f}}{2 \pi} \gamma_{f}^{\left(j_{2}\right)}\right)
$$

Miller [Mil02] observed that an advantage of studying the 2-level density is that, even for arbitrarily small support, the three orthogonal types of symmetry are mutually distinguishable (see [Mil04] where it is used to discern the symmetry group of families of elliptic curves). An analogous definition holds for the $n$-level density; as the signs of our families are constant, our arguments can easily be extended to determining the $n$-level density (although the support decreases with $n$ ).

By [KS99a], the $n$-level densities for the classical compact groups are

$$
\begin{aligned}
W_{n, \mathrm{SO}(\text { even })}(x) & =\operatorname{det}\left(K_{1}\left(x_{i}, x_{j}\right)\right)_{i, j \leqslant n}, \\
W_{n, \mathrm{SO}(\text { odd })}(x) & =\operatorname{det}\left(K_{-1}\left(x_{i}, x_{j}\right)\right)_{i, j \leqslant n}+\sum_{k=1}^{n} \delta\left(x_{k}\right) \operatorname{det}\left(K_{-1}\left(x_{i}, x_{j}\right)\right)_{i, j \neq k}, \\
W_{n, \mathrm{O}}(x) & =\frac{1}{2} W_{n, \mathrm{SO}(\text { even })}(x)+\frac{1}{2} W_{n, \mathrm{SO}(\text { odd })}(x), \\
W_{n, \mathrm{U}}(x) & =\operatorname{det}\left(K_{0}\left(x_{i}, x_{j}\right)\right)_{i, j \leqslant n}, \\
W_{n, \mathrm{USp}}(x) & =\operatorname{det}\left(K_{-1}\left(x_{i}, x_{j}\right)\right)_{i, j \leqslant n},
\end{aligned}
$$

where $K(y)=\sin \pi y / \pi y, K_{\epsilon}(x, y)=K(x-y)+\epsilon K(x+y)$ for $\epsilon=0, \pm 1$, and $\delta(x)$ is the Dirac delta functional; see [HM06] for a more tractable formula for the $n$th centered moments for test functions whose Fourier transforms have support suitably restricted. It is often more convenient to work with the Fourier transforms of the densities. For the 1-level densities, we have

$$
\begin{aligned}
\widehat{W}_{1, \mathrm{SO}(\text { even })}(u) & =\delta(u)+\frac{1}{2} I(u), \\
\widehat{W}_{1, \mathrm{SO}(\text { odd })}(u) & =\delta(u)-\frac{1}{2} I(u)+1, \\
\widehat{W}_{1, \mathrm{O}}(u) & =\delta(u)+\frac{1}{2}, \\
\widehat{W}_{1, \mathrm{U}}(u) & =\delta(u), \\
\widehat{W}_{1, \mathrm{USp}}(u) & =\delta(u)-\frac{1}{2} I(u),
\end{aligned}
$$

where $I(u)$ is the characteristic function of $[-1,1]$. The three orthogonal densities are indistinguishable for test functions of small support. Explicitly, for test functions $g$ such that $\operatorname{supp}(\widehat{g}) \subset(-1,1)$, 
we have

$$
\begin{aligned}
\int \widehat{g}(u) \widehat{W}_{1, \mathrm{SO}(\text { even })}(u) d u & =\widehat{g}(u)+\frac{1}{2} g(0), \\
\int \widehat{g}(u) \widehat{W}_{1, \mathrm{SO}(\text { odd })}(u) d u & =\widehat{g}(u)+\frac{1}{2} g(0), \\
\int \widehat{g}(u) \widehat{W}_{1, \mathrm{O}}(u) d u & =\widehat{g}(u)+\frac{1}{2} g(0), \\
\int \widehat{g}(u) \widehat{W}_{1, \mathrm{U}}(u) d u & =\widehat{g}(u), \\
\int \widehat{g}(u) \widehat{W}_{1, \mathrm{USp}}(u) d u & =\widehat{g}(u)-\frac{1}{2} g(0) .
\end{aligned}
$$

We record the effect of the Fourier transform of the 2-level density kernel on our test functions. Let $c(\mathcal{G})=0$ (respectively, $\left.\frac{1}{2}, 1\right)$ for $\mathcal{G}=\mathrm{SO}$ (even) (respectively, $\mathrm{O}, \mathrm{SO}($ odd) $)$. For even functions $\widehat{g}_{1}\left(u_{1}\right) \widehat{g}_{2}\left(u_{2}\right)$ supported in $\left|u_{1}\right|+\left|u_{2}\right|<1$,

$$
\begin{aligned}
\iint \widehat{g_{1}}\left(u_{1}\right) \widehat{g_{2}}\left(u_{2}\right) \widehat{W_{2, \mathcal{G}}}(u) d u_{1} d u_{2}= & {\left[\widehat{g}_{1}(0)+\frac{1}{2} g_{1}(0)\right]\left[\widehat{g}_{2}(0)+\frac{1}{2} g_{2}(0)\right]+2 \int|u| \widehat{g}_{1}(u) \widehat{g}_{2}(u) d u } \\
& -2 \widehat{g_{1} g_{2}}(0)-g_{1}(0) g_{2}(0)+c(\mathcal{G}) g_{1}(0) g_{2}(0) .
\end{aligned}
$$

Thus, for arbitrarily small support, the 2-level density distinguishes between the three orthogonal groups (see [Mil02] for the calculation).

\subsection{Cusp forms}

We quickly review some facts about cusp forms; see [Iwa97, ILS00] for details. Let $S_{k}$ be the space of holomorphic cusp forms of weight $k$ (an even positive integer) and level 1 (that is, for the full modular group $\Gamma=\mathrm{SL}(2, \mathbb{Z}))$. Let $H_{k}$ be a basis of Hecke eigenforms. Then

$$
\operatorname{dim} S_{k}=\left|H_{k}\right|=\frac{k}{12}+O(1)
$$

Any $f \in H_{k}$ has a Fourier expansion

$$
f(z)=\sum_{n=1}^{\infty} a_{f}(n) e(n z),
$$

and we shall henceforth assume that $f$ is normalized so that $a_{f}(1)=1$. Two other useful normalizations for the coefficients are

$$
\begin{aligned}
& \lambda_{f}(n)=a_{f}(n) n^{-(k-1) / 2} \\
& \psi_{f}(n)=\sqrt{\frac{\Gamma(k-1)}{(4 \pi n)^{k-1}}} \frac{1}{\|f\|} a_{f}(n),
\end{aligned}
$$

with $\|f\|$ the Petersson $L^{2}$-norm of $f$. As mentioned in Remark 2.1, $\psi_{f}(n)$ will lead to a weighted sum which simplifies the application of the Petersson formula. The multiplicativity properties of the Fourier coefficients will be essential in our investigations.

Lemma 2.2. Let $f$ be a cuspidal Hecke eigenform of level 1. Then

$$
\lambda_{f}(m) \lambda_{f}(n)=\sum_{d \mid(m, n)} \lambda_{f}\left(\frac{m n}{d^{2}}\right) .
$$

In particular, we have the following corollary. 


\section{E. Dueñez ANd S. J. Miller}

Corollary 2.3. Let $(m, n)=1$, and $p$ be a prime. Then

$$
\begin{aligned}
\lambda_{f}(m) \lambda_{f}(n) & =\lambda_{f}(m n) \\
\lambda_{f}(p)^{2} & =\lambda_{f}\left(p^{2}\right)+1 .
\end{aligned}
$$

\subsection{Summation formulas}

We recall some standard formulas for summing Fourier coefficients over our families and test functions over primes.

DeFinition 2.4. Let

$$
\begin{aligned}
\Delta_{k}(m, n) & =\sum_{f \in H_{k}} \psi_{f}(m) \bar{\psi}_{f}(n) \\
\delta(m, n) & = \begin{cases}1 & \text { if } m=n \\
0 & \text { otherwise }\end{cases}
\end{aligned}
$$

We rephrase the results from [ILS00] in our language. By their equations (2.8), (2.52)-(2.54), and recalling that $\left|H_{k}\right|=k / 12+O(1)$, we find

$$
\Delta_{k}(m, n)=\frac{\zeta(2)}{\left|H_{k}\right|+O(1)} \sum_{f \in H_{k}} \frac{\lambda_{f}(m) \lambda_{f}(n)}{L\left(1, \operatorname{sym}^{2} f\right)} .
$$

Lemma 2.5 (Petersson formula). For $(m, n)=1, m$ and $n$ of at most $b$ factors,

$$
\Delta_{k}(m, n)=\delta(m, n)+O_{b}\left(\frac{m^{1 / 4} n^{1 / 4} \log m n}{k^{5 / 6}}\right) .
$$

For $m, n$ as above and $12 \pi \sqrt{m n} \leqslant k$,

$$
\Delta_{k}(m, n)=\delta(m, n)+O\left(\frac{\sqrt{m n}}{2^{k}}\right) .
$$

Note that (2.16) and (2.17) are Corollaries 2.2 and 2.3 of [ILS00].

The Petersson formula allows us to easily evaluate certain weighted sums of the Fourier coefficients. As $\|f\|$ is related to $L\left(1, \operatorname{sym}^{2} f\right)$, the natural weights are the harmonic weights $\omega_{f}=$ $\zeta(2) / L\left(1, \phi \times \operatorname{sym}^{2} f\right)$. These weights are almost constant (see (3.34)), and following [ILS00] we may remove these weights in the applications below. See $\S 3.2 .1$ for more details. We call terms with $m=n$ diagonal terms; the remaining terms are called non-diagonal. For small support, the non-diagonal terms will not contribute.

Remark 2.6. There exist explicit formulas, involving Bessel functions and Kloosterman sums, for the error terms in the expansion of $\Delta_{k}(m, n)$. For the families studied in [ILS00], by analyzing these terms' contributions they are able to work with test functions with support greater than $[-1,1]$, and hence distinguish $\mathrm{SO}$ (even) from $\mathrm{SO}$ (odd); see also [HM06] where these terms are handled for the $n$-level densities. Increasing the support has applications to non-vanishing results at the central point. We will not be able to obtain such large support for our families; however, by studying the 2-level density, we can still distinguish $\mathrm{SO}$ (even) from $\mathrm{SO}$ (odd).

The following are immediate applications of the prime number theorem. 
Lemma 2.7. Let $\widehat{F}$ be an even Schwartz function of compact support. Then for any positive integer $a$,

$$
\begin{aligned}
& \sum_{p} \widehat{F}\left(a \frac{\log p}{\log R}\right) \frac{\log p}{\log R} \frac{1}{p}=\frac{1}{2 a} F(0)+O\left(\frac{1}{\log R}\right) \\
& \sum_{p} \widehat{F}\left(\frac{\log p}{\log R}\right) \frac{4 \log ^{2} p}{\log ^{2} R} \frac{1}{p}=2 \int_{-\infty}^{\infty}|u| \widehat{F}(u) d u+O\left(\frac{1}{\log R}\right) .
\end{aligned}
$$

\section{The family $\mathcal{F}_{\phi \times \mathrm{sym}^{2} H_{k}}=\left\{\phi \times \mathrm{sym}^{2} f: f \in H_{k}\right\}$}

We provide evidence that the underlying symmetry of the family $\mathcal{F}_{\phi \times \mathrm{sym}^{2} H_{k}}=\left\{\phi \times \mathrm{sym}^{2} f: f \in H_{k}\right\}$ is $\mathrm{SO}$ (even). In $\S 3.1$ we calculate the required quantities to investigate the distribution of the lowlying zeros. In $\S 3.2$ we calculate the one-level density for test functions whose Fourier transform has small support, proving the first half of Theorem 1.2. Although the support is contained in $(-1,1)$, the evidence is enough to discard the possibility of symplectic (or unitary) symmetry; however, the 1-level density in this range cannot distinguish between $\mathrm{O}, \mathrm{SO}$ (even), and $\mathrm{SO}$ (odd) (even though the even functional equations suggest, of course, that $\mathrm{SO}$ (even) is the type). To rectify this deficiency, in $\S 3.3$ we calculate the 2-level density for small support; this suffices to eliminate $\mathrm{O}$ and $\mathrm{SO}$ (odd) and will complete the proof of Theorem 1.2.

\subsection{Definition, gamma factors, and the functional equation}

We use the notation of $\S 2.2$ for the holomorphic Hecke eigenform $f$ and its Hecke eigenvalues $\lambda_{f}(n)$. Let $\phi$ be a fixed even Hecke-Maass cuspidal eigenform with Laplacian eigenvalue $\lambda_{\phi}=\frac{1}{4}+t_{\phi}^{2}$ for the full modular group $\Gamma=\operatorname{SL}(2, \mathbb{Z})$. We normalize $\phi$ so that $a_{\phi}(1)=1$, and denote by $\lambda_{\phi}(n)$ the corresponding Hecke eigenvalues.

For any unramified prime $p$, the Satake parameters (of the principal-series representation of $\left.\mathrm{GL}_{2}\left(\mathbb{Q}_{p}\right)\right)$ associated to $f$ are two complex numbers $\alpha_{p}, \tilde{\alpha}_{p}=\alpha_{p}^{-1}$ satisfying

$$
\lambda_{f}\left(p^{\nu}\right)=\sum_{\ell=1}^{\nu} \alpha_{p}^{\ell} \tilde{\alpha}_{p}^{\nu-\ell}
$$

Since $f$ is of level 1, every prime is unramified. By the work of Deligne, $\left|\alpha_{p}\right|=1$ (the local representation is tempered) so that in fact $\tilde{\alpha}_{p}$ is the complex conjugate $\bar{\alpha}_{p}$ of $\alpha_{p}$. Thus, $\lambda_{f}(p)=\alpha_{p}+\alpha_{p}^{-1}$ alone determines $\alpha_{p}, \alpha_{p}^{-1}$. By (3.1), all of the $\lambda_{f}\left(p^{\nu}\right)$ are algebraically expressible in terms of $\lambda_{f}(p)$ (formula (3.1) is indeed equivalent to the multiplicativity of the Fourier coefficients).

The Maass form $\phi$ has Satake parameters $\beta_{p}, \tilde{\beta}_{p}=\beta_{p}^{-1}$. The Ramanujan conjecture states that $\left|\beta_{p}\right|=1$; while this is still open for Maass forms, powerful bounds towards Ramanujan are available. Kim and Shahidi [KS02] proved the crucial (for us) bound $\left|\beta_{p}\right|,\left|\tilde{\beta}_{p}\right| \leqslant p^{5 / 34}$. Observe that $\frac{5}{34}<\frac{1}{6}$, which has many important consequences (see $[\mathrm{KS} 02, \S 8]$ ), and is perhaps not coincidentally all we need below. The exponent has been recently improved by Kim and Sarnak to $\frac{7}{64}$ (see [Kim03, Appendix Two]).

Denote by $\operatorname{sym}^{2} f$ the Gelbart-Jacquet (symmetric-square) lift to (an automorphic cuspidal representation of) GL(3) of the cusp form $f$ (see [GJ78]). Its Fourier coefficients are [Bum84, Bum89]

$$
a_{\mathrm{sym}^{2} f}\left(m_{1}, m_{2}\right)=\sum_{d \mid\left(m_{1}, m_{2}\right)} \lambda_{\mathrm{sym}^{2} f}\left(\frac{m_{1}}{d}, 1\right) \lambda_{\mathrm{sym}^{2} f}\left(\frac{m_{2}}{d}, 1\right) \mu(d),
$$

where $\mu$ is the Möbius function and

$$
\lambda_{\mathrm{sym}^{2} f}(r, 1)=\sum_{s^{2} t=r} \lambda_{f}\left(t^{2}\right) .
$$




\section{E. Dueñez ANd S. J. Miller}

The symmetric-square $L$-function of $f$ is then

$$
L\left(s, \operatorname{sym}^{2} f\right)=\sum_{m=1}^{\infty} \lambda_{\operatorname{sym}^{2} f}(m, 1) m^{-s} .
$$

If, as before, $\alpha_{p}, \alpha_{p}^{-1}$ are the Satake parameters of $f$, then the parameters $\sigma_{p}(j)(j=1,2,3)$ of $\operatorname{sym}^{2} f$ at any prime $p$ are the numbers $\alpha_{p}^{2}, 1, \alpha_{p}^{-2}$.

Denoting by $\lambda_{\phi}(r)$ the $r$ th Hecke eigenvalue of $\phi$, the Rankin-Selberg convolution $L\left(s, \phi \times \operatorname{sym}^{2} f\right)$ is the Dirichlet series

$$
\begin{aligned}
L\left(s, \phi \times \operatorname{sym}^{2} f\right) & =\sum_{m_{1}, m_{2} \geqslant 1} \lambda_{\phi}\left(m_{1}\right) \lambda_{f}\left(m_{2}\right) a_{F}\left(m_{1}, m_{2}\right)\left(m_{1} m_{2}^{2}\right)^{-s} \\
& =\sum_{m} \lambda_{\phi, \operatorname{sym}^{2} f}(m) m^{-s},
\end{aligned}
$$

where

$$
\lambda_{\phi \times \operatorname{sym}^{2} f}(m)=\sum_{m_{1} m_{2}^{2}=m} \lambda_{\phi}\left(m_{1}\right) \lambda_{f}\left(m_{2}\right) a_{F}\left(m_{1}, m_{2}\right) .
$$

In fact, also by the work of Kim and Shahidi [KS02] (and the appendix by Bushnell and Henniart), $L\left(s, \phi \times \operatorname{sym}^{2} f\right)$ is an automorphic $L$-function $L(s, \pi)$ (for a suitable automorphic representation $\pi$ of GL(6).) This ensures the standard properties (entire of order one, bounded in vertical strips, and functional equation) for $L\left(s, \phi \times \operatorname{sym}^{2} f\right)$. In particular, $L\left(s, \phi \times \operatorname{sym}^{2} f\right)$ conjecturally satisfies the Riemann hypothesis in the usual sense: $L\left(s, \phi \times \operatorname{sym}^{2} f\right)=0$ and $0 \leqslant \Im s \leqslant 1$ implies $\Im s=\frac{1}{2}$.

The Satake parameters $\delta_{p}(j)(j=1, \ldots, 6)$ of $\pi_{p}$ are the six numbers $\alpha_{p}^{ \pm 2} \beta_{p}^{ \pm 1}$ and $\beta_{p}^{ \pm 1}$. Furthermore, each $L\left(s, \phi \times \operatorname{sym}^{2} f\right)$ has an even functional equation. The proof of this assertion is given in Appendix A.

For $\Re s$ large, the logarithmic derivative of $L\left(s, \phi \times \operatorname{sym}^{2} f\right)$ is given by the Dirichlet series

$$
\frac{L^{\prime}}{L}\left(s, \phi \times \operatorname{sym}^{2} f\right)=\sum_{m=0}^{\infty} \Lambda(m) a_{\phi \times \operatorname{sym}^{2} f}(m) m^{-s},
$$

where $\Lambda(m)$ is von Mangoldt's function and

$$
a_{\phi \times \operatorname{sym}^{2} f}\left(p^{\nu}\right)=\sum_{j=1}^{6} \delta_{p}(j)^{\nu} .
$$

Define now the archimedean (gamma) factor

$$
\begin{aligned}
L_{\infty}\left(s, \phi, \operatorname{sym}^{2} f\right):= & \Gamma_{\mathbb{R}}\left(s+k-1+i t_{\phi}\right) \Gamma_{\mathbb{R}}\left(s+k-1-i t_{\phi}\right) \\
& \times \Gamma_{\mathbb{R}}\left(s+k+i t_{\phi}\right) \Gamma_{\mathbb{R}}\left(s+k-i t_{\phi}\right) \Gamma_{\mathbb{R}}\left(s+1+i t_{\phi}\right) \Gamma_{\mathbb{R}}\left(s+1-i t_{\phi}\right),
\end{aligned}
$$

where

$$
\Gamma_{\mathbb{R}}(s):=\pi^{-s / 2} \Gamma\left(\frac{s}{2}\right)
$$

The completed $L$-function

$$
\Lambda\left(s, \phi \times \operatorname{sym}^{2} f\right):=L_{\infty}\left(s, \phi, \operatorname{sym}^{2} f\right) L\left(s, \phi \times \operatorname{sym}^{2} f\right)
$$

for (3.5) satisfies the functional equation

$$
\Lambda\left(s, \phi \times \operatorname{sym}^{2} f\right)=\Lambda\left(1-s, \phi \times \operatorname{sym}^{2} f\right) .
$$

As the functional equation is even, we expect to observe either $\mathrm{SO}$ (even) or symplectic symmetry. 


\section{The LOW LYING ZEROS OF TWO FAMILIES OF $L$-FUNCTIONS}

Following Rudnick and Sarnak [RS96], we define the six archimedean parameters $\mu_{j}(j=$ $1, \ldots, 6)$ by the requirement that $\frac{1}{2}+\mu_{j}$ is either

$$
k \pm \frac{1}{2} \pm i t_{\phi} \quad \text { or } \quad \frac{3}{2} \pm i t_{\phi}
$$

3.1.1 Explicit formula. A smooth form of the explicit formula for $L\left(s, \phi \times \operatorname{sym}^{2} f\right)$ is as follows (see [RS96] for a proof). Let $g \in C_{c}^{\infty}(\mathbb{R})$ be an even Schwartz function whose Fourier transform

$$
\hat{g}(y)=\int_{-\infty}^{\infty} g(x) e^{-2 \pi i x y} d x
$$

is compactly supported. Let $R>0$ and write the non-trivial zeros of $L\left(s, \phi \times \operatorname{sym}^{2} f\right)$ as $\rho_{j}=\frac{1}{2}+i \gamma_{j}$; we have $j \in \mathbb{Z}-\{0\}$ as the functional equation is even. Note $\gamma_{j} \in \mathbb{R}$ is equivalent to the GRH. Then

$$
\sum_{j} g\left(\frac{\gamma_{j}}{2 \pi} \log R\right)=\frac{A}{\log R}-2 \sum_{p} \sum_{\nu=1}^{\infty} \hat{g}\left(\frac{\nu \log p}{\log R}\right) \frac{a_{\phi \times \operatorname{sym}^{2} f}\left(p^{\nu}\right) \log p}{p^{\nu / 2} \log R},
$$

where

$$
A=\int_{-\infty}^{\infty} \sum_{j=1}^{6}\left(\frac{\Gamma_{\mathbb{R}}^{\prime}}{\Gamma_{\mathbb{R}}}\left(\mu_{j}+\frac{1}{2}+\frac{2 \pi i x}{\log R}\right)+\frac{\Gamma_{\mathbb{R}}^{\prime}}{\Gamma_{\mathbb{R}}}\left(\overline{\mu_{j}}+\frac{1}{2}+\frac{2 \pi i x}{\log R}\right)\right) g(x) d x
$$

3.1.2 Gamma factor contribution. Recall $\Gamma_{\mathbb{R}}(s)=\pi^{-s / 2} \Gamma(s / 2)$. Thus

$$
\frac{\Gamma_{\mathbb{R}}^{\prime}(s)}{\Gamma_{\mathbb{R}}(s)}=-\frac{\log \pi}{2}+\frac{1}{2} \frac{\Gamma^{\prime}(s / 2)}{\Gamma(s / 2)} .
$$

Let $r=2 \pi x / \log R$. Then the sum in (3.16) is equal to

$$
-6 \log \pi+\frac{1}{2} \sum_{j=1}^{6}\left[\frac{\Gamma^{\prime}}{\Gamma}\left(\frac{1}{4}+\frac{\mu_{j}}{2}+\frac{i r}{2}\right)+\frac{\Gamma^{\prime}}{\Gamma}\left(\frac{1}{4}+\frac{\overline{\mu_{j}}}{2}-\frac{i r}{2}\right)\right],
$$

where $\mu_{j}=k \pm \frac{1}{2} \pm i t_{\phi}$ (for four values) and $\frac{3}{2} \pm i t_{\phi}$ (for the other two). We use (see [ILS00] or [GR65, formula 8.363.3]) that for $a, b \in \mathbb{R}, a>0$,

$$
\frac{\Gamma^{\prime}}{\Gamma}(a+b i)+\frac{\Gamma^{\prime}}{\Gamma}(a-b i)=2 \frac{\Gamma^{\prime}}{\Gamma}(a)+O\left(a^{-2} b^{2}\right),
$$

and for $\alpha \geqslant \frac{1}{4}$,

$$
\frac{\Gamma^{\prime}}{\Gamma}\left(\alpha+\frac{1}{4}\right)=\log \alpha+O(1)
$$

Thus, in the $\Gamma^{\prime} / \Gamma$ factors, the $\mu_{j}=\frac{3}{2} \pm i t_{\phi}$ terms are $O(1)$ with respect to $k$. Set $a_{+}=\frac{1}{2}$ and $a_{-}=0$. Matched in complex-conjugate pairs, the other eight terms give

$$
\begin{gathered}
\frac{\Gamma^{\prime}}{\Gamma}\left(\frac{k}{2}+a_{ \pm}+i\left( \pm \frac{t_{\phi}}{2}+r\right)\right)+\frac{\Gamma^{\prime}}{\Gamma}\left(\frac{k}{2}+a_{ \pm}-i\left( \pm \frac{t_{\phi}}{2}+r\right)\right) \\
=2 \frac{\Gamma^{\prime}}{\Gamma}\left(\frac{k}{2}+a_{ \pm}\right)+O\left(\frac{\left|t_{\phi}\right|^{2}+r^{2}}{k^{2}}\right)
\end{gathered}
$$

and

$$
\frac{\Gamma^{\prime}}{\Gamma}\left(\frac{k}{2}+a_{ \pm}\right)=\log \left(\frac{k}{2}+a_{ \pm}-\frac{1}{4}\right)+O(1)=\log k+O(1) .
$$

Note for $k \geqslant 2$, the condition of having the argument greater than $\frac{1}{4}$ is trivially met. The main term in the sum in (3.16) is simply $\frac{1}{2} \cdot 4 \cdot 2 \log k=4 \log k$. The main contribution of the term $A / \log R$ 


\section{E. Dueñez And S. J. Miller}

in $(3.15)$ is

$$
\frac{4 \log k}{\log R} \int_{-\infty}^{\infty} g(x) d x=\frac{\log k^{4}}{\log R} \cdot \hat{g}(0)
$$

In the $k$-aspect, $\phi \times \operatorname{sym}^{2} f$ looks like a GL(4) object. A natural choice for the analytic conductors is therefore $k^{4}$. With this scaling of the zeros, the test function on the the left-hand side of (3.23) is evaluated at points which have mean average spacing of one near the central point (Riemann's classical critical zero-counting formula). As the quotient depends only on the logarithm of the conductor, as $k \rightarrow \infty$ the choice of any fixed constant multiple of $k^{4}$ for the conductor will give the same answer (see [ILS00]). We have proved the following lemma.

Lemma 3.1. For $L\left(s, \phi \times \mathrm{sym}^{2} f\right)$, up to lower order terms the contribution from the $\Gamma$-factors in the explicit formula equals $\widehat{g}(0)$, and the analytic conductor is equal to $k^{4}$. Assuming the GRH, the non-trivial zeros of $L\left(s, \phi \times \operatorname{sym}^{2} f\right)$ are $\frac{1}{2}+i \gamma_{\phi \times \operatorname{sym}^{2} f}^{(j)}$ with $\gamma_{\phi \times \operatorname{sym}^{2} f}^{(j)} \in \mathbb{R}$. Taking $R=k^{4}$, the explicit formula (3.15) becomes

$$
\sum_{j} g\left(\frac{\gamma_{\phi \times \mathrm{sym}^{2} f}^{(j)}}{2 \pi} \log R\right)=\widehat{g}(0)-2 \sum_{p} \sum_{\nu=1}^{\infty} \hat{g}\left(\frac{\nu \log p}{\log R}\right) \frac{a_{\phi \times \mathrm{sym}^{2} f}\left(p^{\nu}\right) \log p}{p^{\nu / 2} \log R}+O\left(\frac{1}{\log R}\right)
$$

The appearance of the term $\widehat{g}(0)=\int g(x) d x$ on the right-hand side of (3.24) naturally corresponds to the (expected) term $\int \widehat{g}(\xi) \delta(\xi) d \xi$ due to the delta mass at the origin in the Fourier transform of the 1-level density (see (2.6)). The second term (double sum) above will eventually be matched to $\int \widehat{g}(\xi) \frac{1}{2} \eta(\xi) d \xi$, and this will exclude symplectic as a possibility.

Remark 3.2. It is fortunate for us that the analytic conductors of $L\left(s, \phi \times \operatorname{sym}^{2} f\right)$ depend weakly on $f$. Specifically, as the only dependence on $f$ is through its weight $k$, one scaling works for all elements of our family. Oscillating conductors in a family can sometimes be handled (one recourse is to use the average log conductor as in [Sil98, You06, DM06]; another approach is a more careful analysis and sieving, as in [Mil04] where the conductors are monotone).

3.1.3 Relation of $a_{\phi \times \mathrm{sym}^{2} f}$ to $\lambda_{f}$ and $\lambda_{\phi}$. To evaluate the double sum in (3.24), we express $a_{\phi \times \operatorname{sym}^{2} f}\left(p^{\nu}\right)$ in terms of $\lambda_{f}, \lambda_{\phi}$. Note that

$$
\begin{aligned}
a_{\phi \times \operatorname{sym}^{2} f}\left(p^{\nu}\right) & =\alpha_{p}^{2 \nu} \beta_{p}^{\nu}+\alpha_{p}^{-2 \nu} \beta_{p}^{\nu}+\alpha_{p}^{2 \nu} \beta_{p}^{-\nu}+\alpha_{p}^{-2 \nu} \beta_{p}^{-\nu}+\beta_{p}^{\nu}+\beta_{p}^{-\nu} \\
& =\left(\alpha_{p}^{2 \nu}+1+\alpha_{p}^{-2 \nu}\right)\left(\beta_{p}^{\nu}+\beta_{p}^{-\nu}\right) .
\end{aligned}
$$

Case $\nu=1$. We have

$$
a_{\phi \times \operatorname{sym}^{2} f}(p)=\left(\alpha_{p}^{2}+1+\alpha_{p}^{-2}\right)\left(\beta_{p}+\beta_{p}^{-1}\right)=\lambda_{f}\left(p^{2}\right) \lambda_{\phi}(p) .
$$

Case $\nu \geqslant 2$. We have

$$
\alpha_{p}^{2 \nu}+1+\alpha_{p}^{-2 \nu}=\left(\alpha_{p}^{2 \nu}+\alpha_{p}^{2(\nu-1)}+\cdots+\alpha_{p}^{-2(\nu-1)}+\alpha_{p}^{-2 \nu}\right)-\left(\alpha_{p}^{2(\nu-1)}+\cdots+\alpha_{p}^{-2(\nu-1)}\right)+1
$$

and

yielding

$$
\beta_{p}^{\nu}+\beta_{p}^{-\nu}=\beta_{p}^{\nu}+\beta_{p}^{\nu-2}+\cdots+\beta_{p}^{-(\nu-2)}+\beta_{p}^{-\nu}-\left(\beta_{p}^{\nu-2}+\cdots+\beta_{p}^{-(\nu-2)}\right)
$$

$$
\begin{aligned}
a_{\phi \times \operatorname{sym}^{2} f}\left(p^{\nu}\right) & =\left(\alpha_{p}^{2 \nu}+1+\alpha_{p}^{-2 \nu}\right)\left(\beta_{p}^{\nu}+\beta_{p}^{-\nu}\right) \\
& =\left(\lambda_{f}\left(p^{2 \nu}\right)-\lambda_{f}\left(p^{2(\nu-1)}\right)+1\right)\left(\lambda_{\phi}\left(p^{\nu}\right)-\lambda_{\phi}\left(p^{\nu-2}\right)\right) .
\end{aligned}
$$

Of course, $\lambda_{\phi}\left(p^{\nu-2}\right)=1$ when $\nu=2$. 


\section{The LOW LYING ZEROS OF TWO FAMILIES OF $L$-FUnCTIONS}

3.1.4 Summary. We have shown the following.

LEMMA 3.3. We have

$$
\begin{aligned}
a_{\phi \times \operatorname{sym}^{2} f}(p) & =\lambda_{\phi}(p) \lambda_{f}\left(p^{2}\right) \\
a_{\phi \times \operatorname{sym}^{2} f}\left(p^{2}\right) & =\left(\lambda_{\phi}\left(p^{2}\right)-1\right) \cdot\left(\lambda_{f}\left(p^{4}\right)-\lambda_{f}\left(p^{2}\right)+1\right) .
\end{aligned}
$$

As we shall see below, the single term ' -1 ' in the first factor of (3.31) is responsible for flipping the symmetry from symplectic (for the $\left\{\operatorname{sym}^{2} f\right\}$ family of [ILS00] which had $a_{\mathrm{sym}^{2} f}=\lambda_{f}\left(p^{4}\right)-\lambda_{f}\left(p^{2}\right)+1$ ) to $\mathrm{SO}$ (even) (for the $\left\{\phi \times \mathrm{sym}^{2} f\right\}$ family we are considering). This behavior is described in more detail in [DM06].

Using the results from Kim and Sarnak [Kim03], we have $\left|\beta_{p}^{ \pm 1}\right| \leqslant p^{7 / 64}$. Since $\left|\alpha_{p}\right| \leqslant 1$, equation (3.8) yields

$$
a_{\phi \times \operatorname{sym}^{2} f}\left(p^{\nu}\right)=\left(\beta_{p}^{\nu}+\beta_{p}^{-\nu}\right) \cdot\left(\alpha_{p}^{2 \nu}+\alpha_{p}^{-2 \nu}+1\right) \ll p^{7 \nu / 64} .
$$

Therefore

$$
\frac{a_{\phi \times \operatorname{sym}^{2} f}\left(p^{\nu}\right)}{p^{\nu / 2}} \ll p^{-25 \nu / 64} .
$$

This immediately implies the following lemma.

Lemma 3.4. The contribution from terms with $\nu \geqslant 3$ in (3.24) can be absorbed into the error term.

Remark 3.5. We do not need the full strength of $\left|\beta_{p}^{ \pm 1}\right| \ll p^{7 / 64}$; any exponent less than $\frac{1}{6}$ suffices. Without such a bound, we would later need to obtain cancellation when averaging the Fourier coefficients over the family (a result of this nature is significantly weaker than proving bounds towards Ramanujan, and follows from the Petersson formula).

\subsection{1-level density}

As $\mathcal{F}_{\phi \times \mathrm{sym}^{2} H_{k}}=\left\{\phi \times \operatorname{sym}^{2} f, f \in H_{k}\right\}$, we have $\left|\mathcal{F}_{\phi \times \mathrm{sym}^{2} H_{k}}\right|=\left|H_{k}\right|$. For each $L$-function from $\mathcal{F}_{\phi \times \mathrm{sym}^{2} H_{k}}$ we calculate the 1-level density for its low-lying zeros via the explicit formula; we then average over the family $\mathcal{F}_{\phi \times \operatorname{sym}^{2} H_{k}}$.

3.2.1 Preliminaries. Let $g$ be an even Schwartz function with $\operatorname{supp}(\widehat{g}) \subset(-\sigma, \sigma)$. Following Iwaniec, Luo, and Sarnak [ILS00] or Royer [Roy01], we consider a weighted average over the family $\mathcal{F}_{\phi \times \mathrm{sym}^{2} H_{k}}$ of the expressions (3.24). The weight factors that we use are $\omega_{f}=\zeta(2) / L\left(1, \phi \times \mathrm{sym}^{2} f\right)$. These are positive (by the GRH for $L\left(s, \operatorname{sym}^{2} f\right)$ ), slowly varying, and satisfy

$$
k^{-\epsilon} \ll_{\epsilon} \frac{1}{L\left(1, \mathrm{sym}^{2} f\right)} \ll_{\epsilon} k^{\epsilon}
$$

and

$$
\frac{1}{\left|H_{k}\right|} \sum_{f \in H_{k}} \frac{\zeta(2)}{L\left(1, \operatorname{sym}^{2} f\right)}=1+O\left(\frac{1}{k}\right) .
$$

To simplify the application of the Petersson formula, we have introduced the slowly varying weights $\zeta(2) / L\left(1\right.$, sym $\left.^{2} f\right)$; arguing along the lines of [ILS00] allows one to remove these weights at no cost. We have chosen to leave in the weights in order to emphasize the features of this GL(6) family.

By the GRH, we may denote the non-trivial zeros of $L\left(s, \phi \times \operatorname{sym}^{2} f\right)$ by $\frac{1}{2}+i \gamma_{\phi \times \mathrm{sym}^{2} f}^{(j)}$ with $\gamma_{\phi \times \operatorname{sym}^{2} f}^{(j)} \in \mathbb{R}$. Let $R=k^{4}$. All $L\left(s, \phi \times \operatorname{sym}^{2} f\right)$ have the same analytic conductor, which up to lower-order terms is $k^{4}$. Averaging (3.24) by incorporating the weights and using Lemma 3.4 


\section{E. Dueñez And S. J. Miller}

to absorb the $\nu \geqslant 3$ terms into the error shows that the 1-level density for the family $\mathcal{F}_{\phi \times \mathrm{sym}^{2} H_{k}}$ is

$$
\begin{aligned}
& D_{1, \mathcal{F}_{\phi \times \mathrm{sym}^{2} H_{k}}}(g) \\
& \quad=\frac{1}{\left|H_{k}\right|} \sum_{f \in H_{k}} \frac{\zeta(2)}{L\left(1, \operatorname{sym}^{2} f\right)} \sum_{j} g\left(\gamma_{\phi \times \operatorname{sym}^{2} f}^{(j)} \frac{\log R}{2 \pi}\right) \\
& \quad=\widehat{g}(0)-\frac{2}{\left|H_{k}\right|} \sum_{f \in H_{k}} \frac{1}{L\left(1, \operatorname{sym}^{2} f\right)} \sum_{\nu=1}^{2} \sum_{p=2}^{R^{\sigma}} \frac{a_{\phi \times \operatorname{sym}^{2} f}\left(p^{\nu}\right) \log p}{p^{\nu / 2} \log R} \widehat{g}\left(\nu \frac{\log p}{\log R}\right)+O\left(\frac{1}{\log R}\right) .
\end{aligned}
$$

We are left with analyzing the contribution from the $\nu=1,2$ terms, and comparing this to (2.6). For small support, we will show that there is no contribution from the $\nu=1$ term, and the $\nu=2$ term contributes $\frac{1}{2} g(0)$. This proves the symmetry group is neither unitary nor symplectic. We cannot discard the $\mathrm{O}$ and $\mathrm{SO}$ (odd) symmetries; however, we will be able to eliminate them later by studying the 2-level density.

Remark 3.6. Since Iwaniec, Luo, and Sarnak exclusively use 1-level density arguments, they must use extra averaging to extend their support past $[-1,1]$. By studying the 2-level density we provide compelling evidence for the underlying symmetry being $\mathrm{SO}$ (even) without extra averaging.

3.2.2 Contribution from $\nu=1$. We must evaluate

$$
T_{1}=\frac{1}{\left|H_{k}\right|} \sum_{f \in H_{k}} \frac{\zeta(2)}{L\left(1, \operatorname{sym}^{2} f\right)} \sum_{p=2}^{R^{\sigma}} \frac{a_{\phi \times \mathrm{sym}^{2} f}(p) \log p}{\sqrt{p} \log R} \widehat{g}\left(\frac{\log p}{\log R}\right) .
$$

By Lemma 3.3, $a_{\phi \times \operatorname{sym}^{2} f}(p)=\lambda_{\phi}(p) \lambda_{f}\left(p^{2}\right)$. Since $\lambda_{f}(1)=1$,

$$
T_{1}=\sum_{p=2}^{R^{\sigma}} \frac{\lambda_{\phi}(p) \log p}{\sqrt{p} \log R} \widehat{g}\left(\frac{\log p}{\log R}\right) \frac{\zeta(2)}{\left|H_{k}\right|} \sum_{f \in H_{k}} \frac{\lambda_{f}(1) \lambda_{f}\left(p^{2}\right)}{L\left(1, \operatorname{sym}^{2} f\right)} .
$$

We are led to studying

$$
\frac{\zeta(2)}{\left|H_{k}\right|} \sum_{f \in H_{k}} \frac{\lambda_{f}(1) \lambda_{f}\left(p^{2}\right)}{L\left(1, \operatorname{sym}^{2} f\right)}
$$

We have a non-diagonal term since $p^{2} \neq 1$. By (2.17) of Lemma $2.5, \delta\left(1, p^{2}\right)=0$ and for $p \ll k$ these terms are much less than $p / 2^{k}$. Substituting into the expansion for $T_{1}$, we see that there is no contribution for $R^{\sigma}<k$. Since $R=k^{4}$, this implies that there is no contribution for $\sigma<\frac{1}{4}$. Note that in executing the prime sum, any polynomial bound on $\lambda_{\phi}(p)$ suffices, since the decay in $k$ is exponential.

For primes $p>R^{\frac{1}{4}}$, we cannot use (2.17); instead we use (2.16), which gives much less than $\sqrt{p} \log p / k^{5 / 6}$. This yields a $p$-sum of

$$
\frac{1}{k^{5 / 6}} \sum_{p}^{k^{4 \sigma}} \frac{\log ^{2} p}{\log R} \frac{\lambda_{\phi}(p) \sqrt{p}}{\sqrt{p}}
$$

Let $\delta$ be the best bound towards Ramanujan for $\lambda_{\phi}(p)$; namely, $\lambda_{\phi}(p) \ll p^{\delta}$ (the Ramanujan conjecture is $\delta=0)$. We find that this sum is much less than $k^{4 \sigma(1+\delta)-5 / 6}$; thus, $\sigma<5 / 24(1+\delta)$. Even assuming Ramanujan does not help (this bound is worse than the previous). Thus, (2.17) is better and we obtain that there is no contribution for support up to $\frac{1}{4}$.

Remark 3.7. The reason there is no contribution for small support is that we have a non-diagonal term in the Petersson formula. 


\section{The LOW LYING ZEROS OF TWO FAMILIES OF $L$-FUnCTIONS}

3.2.3 Contribution from $\nu=2$. We must evaluate

$$
T_{2}=-\frac{2}{\left|H_{k}\right|} \sum_{f \in H_{k}} \frac{\zeta(2)}{L\left(1, \operatorname{sym}^{2} f\right)} \sum_{p=2}^{R^{\sigma}} \frac{a_{\phi \times \operatorname{sym}^{2} f}\left(p^{2}\right) \log p}{p \log R} \widehat{g}\left(2 \frac{\log p}{\log R}\right) .
$$

By Lemma 3.3, $a_{\phi \times \operatorname{sym}^{2} f}\left(p^{2}\right)=\left(\lambda_{\phi}\left(p^{2}\right)-1\right) \cdot\left(\lambda_{f}\left(p^{4}\right)-\lambda_{f}\left(p^{2}\right)+1\right)$. Almost all of the terms are non-diagonal. Using $\lambda_{f}(1)=1$ we have the following terms: from $\lambda_{\phi}\left(p^{2}\right)$, we get

$$
\lambda_{\phi}\left(p^{2}\right) \cdot \lambda_{f}(1) \lambda_{f}\left(p^{4}\right), \quad-\lambda_{\phi}\left(p^{2}\right) \cdot \lambda_{f}(1) \lambda_{f}\left(p^{2}\right), \quad \lambda_{\phi}\left(p^{2}\right) \cdot \lambda_{f}(1) \lambda_{f}(1) .
$$

The first two terms are non-diagonal; the Petersson formula yields no contribution for small support. The third term is diagonal. For small support, up to lower-order terms it yields +1 by $(3.35)$ :

$$
\frac{\zeta(2)}{\left|H_{k}\right|} \sum_{f \in H_{k}} \frac{\lambda_{f}(1) \lambda_{f}(1)}{L\left(1, \operatorname{sym}^{2} f\right)}=1+O\left(\frac{1}{k}\right)
$$

This gives

$$
-2 \sum_{p=2}^{R^{\sigma}} \frac{\lambda_{\phi}\left(p^{2}\right) \log p}{p \log R} \widehat{g}\left(2 \frac{\log p}{\log R}\right) .
$$

By the GRH for $L\left(s, \operatorname{sym}^{2} \phi\right)$, this sum is $O(1 / \log R)$ (see [ILS00, $\left.\S 4\right]$ ).

We now handle the three terms from the -1 in the first factor of $a_{\phi \times \operatorname{sym}^{2} f}\left(p^{2}\right)$; these are

$$
-\lambda_{f}(1) \lambda_{f}\left(p^{4}\right), \quad \lambda_{f}(1) \lambda_{f}\left(p^{2}\right), \quad-\lambda_{f}(1) \lambda_{f}(1) .
$$

The first two are non-diagonal, and by the Petersson formula do not contribute for small support. The third term, however, is a diagonal term; up to lower-order corrections, from the Petersson formula its contribution is -1 , and we are left with

$$
2 \sum_{p} \frac{\log p}{p \log R} \widehat{g}\left(2 \frac{\log p}{\log R}\right) .
$$

By Lemma 2.7, the above sum (up to lower-order terms) is $\frac{1}{2} g(0)$.

Therefore, for small support, the $\nu=2$ piece contributes $\frac{1}{2} g(0)+o(1)$, with the main term arising from the sixth term in the expansion of $a_{\phi \times \operatorname{sym}^{2} f}\left(p^{2}\right)$. At this point we have enough evidence to discard the unitary and symplectic symmetries. Since the functional equations in (3.12) are even, this certainly points to the underlying symmetry being $\mathrm{SO}$ (even), but we cannot yet discard the full orthogonal or $\mathrm{SO}$ (odd) symmetries. This will be done in $\S 3.3$.

We now determine how large we may take the support. Of the six pieces which do not contribute to the main term, the worst error term is from $\lambda_{\phi}\left(p^{2}\right) \cdot \lambda_{f}(1) \lambda_{f}\left(p^{4}\right)$. By $(2.17)$, if $1 \cdot p^{4} \ll k^{2}$, the sum over $f \in H_{k}$ is much less than $p^{2} / 2^{k}$. Again, any polynomial bound for $\lambda_{\phi}\left(p^{2}\right)$ yields the sum over primes $p \ll k^{1 / 2}$ is a lower-order term. Since $R=k^{4}$, this yields no contribution for $\sigma<\frac{1}{8}$. Therefore, up to lower-order terms the contribution is $\frac{1}{2} g(0)$ for $\sigma<\frac{1}{8}$.

The reason for the sharp decrease in support (relative to the $\nu=1$ term) is because we have a $\lambda_{f}(1) \lambda_{f}\left(p^{4}\right)$. Another possibility is to use (2.16), which, for the $\lambda_{\phi}\left(p^{2}\right) \cdot \lambda_{f}(1) \lambda_{f}\left(p^{4}\right)$ term, gives

$$
\frac{1}{k^{5 / 6}} \sum_{p}^{k^{4 \sigma}} \frac{\log ^{2} p}{\log R} \frac{\lambda_{\phi}\left(p^{2}\right) \cdot p}{p}
$$




\section{E. Dueñez ANd S. J. Miller}

From (2.13), $\lambda_{\phi}\left(p^{2}\right)=\lambda_{\phi}(p)^{2}-1$. Substituting into (3.47), the -1 does not contribute for $\sigma<\frac{5}{24}$, and we are left with bounding

$$
\frac{1}{k^{5 / 6}} \sum_{p=2}^{k^{4 \sigma}} \frac{\log ^{2} p}{\log R} \lambda_{\phi}(p)^{2} \ll \frac{\log k^{4}}{k^{5 / 6}} \sum_{n=1}^{k^{4 \sigma}}\left|\lambda_{\phi}(n)\right|^{2} .
$$

One could use bounds towards Ramanujan; however, all we need is that Ramanujan holds on average, namely the sum of $\left|\lambda_{\phi}(n)\right|^{2}$ (see $[\operatorname{Iwa95},(8.7)]$ ) is

$$
\sum_{n=1}^{X}\left|\lambda_{\phi}(n)\right|^{2} \ll_{\phi} X
$$

This yields that the sum in (3.48) does not contribute for $\sigma<\frac{5}{24}$.

A similar argument applied to the other terms show none of them contribute as well for such support; one must check that the error term for the $\lambda_{f}(1) \lambda_{f}(1)$ term (which is the diagonal piece responsible for $\left.\frac{1}{2} g(0)\right)$ does not contribute in this range. This completes the proof of the first part of Theorem 1.2.

As $\operatorname{supp}(\widehat{\phi}) \subset(-1,1)$, while every $\Lambda\left(s, \phi \times \operatorname{sym}^{2} f\right)$ has even functional equation, we cannot conclude the symmetry is $\mathrm{SO}$ (even) and not $\mathrm{O}$ or $\mathrm{SO}$ (odd). There are two natural ways to try and increase the support. The first is to average over even Maass forms $\phi$; unfortunately, we would have to let $t_{j}$ grow to a power of $k$, which would change the conductor arguments. Another approach is to average over the weight $k$ (as in the investigation of $\operatorname{sym}^{2} f$ in [ILS00]). By averaging over weight, they triple the support; however, as we start with support less than $\frac{1}{3}$, such methods are insufficient to break $(-1,1)$. We therefore study the 2-level density, which even for arbitrarily small support can distinguish between the three orthogonal groups (see [Mil02, Mil04]).

\subsection{2-level density}

We complete the proof of Theorem 1.2. As in $\S 3.2$, for convenience in applying the Petersson formula we study a weighted 2-level density. Thus (2.3) becomes

$$
D_{2, \mathcal{F}_{\phi \times \mathrm{sym}^{2} H_{k}}}(g)=\frac{1}{\left|H_{k}\right|} \sum_{f \in H_{k}} \frac{\zeta(2)}{L\left(1, \operatorname{sym}^{2} f\right)} \sum_{\substack{j_{1}, j_{2} \\ j_{1} \neq \pm j_{2}}} g_{1}\left(\frac{\log R}{2 \pi} \gamma_{f}^{\left(j_{1}\right)}\right) g_{2}\left(\frac{\log R}{2 \pi} \gamma_{f}^{\left(j_{2}\right)}\right) .
$$

The sum is over zeros $j_{1} \neq \pm j_{2}$. As all functional equations are even and we are assuming the GRH, the zeros occur in complex conjugate pairs; this is the first time the sign of the functional equation enters our arguments. We may rewrite the 2-level expression as a sum over all pairs of zeros, minus twice the sum over all zeros, yielding

$D_{2, \mathcal{F}_{\phi \times \mathrm{sym}^{2} H_{k}}}(g)=\frac{1}{\left|H_{k}\right|} \sum_{f \in H_{k}} \frac{\zeta(2)}{L\left(1, \mathrm{sym}^{2} f\right)} \sum_{j_{1}, j_{2}} g_{1}\left(\frac{\log R}{2 \pi} \gamma_{f}^{\left(j_{1}\right)}\right) g_{2}\left(\frac{\log R}{2 \pi} \gamma_{f}^{\left(j_{2}\right)}\right)-2 D_{1, \mathcal{F}_{\phi \times \mathrm{sym}^{2} H_{k}}}\left(g_{1} g_{2}\right)$.

In the above, the second term is a 1-level density with test function $\left(g_{1} g_{2}\right)(x)$. For small support, we have shown that this term is just $\widehat{g_{1} g_{2}}(0)+\frac{1}{2}\left(g_{1} g_{2}\right)(0)$. A crucial part of the above expansion is that each $\Lambda\left(s, \phi \times \operatorname{sym}^{2} f\right)$ has even sign. This allows us to pair off the zeros, $\gamma_{f}^{(j)}$ and $\gamma_{f}^{(-j)}$. Thus summing over distinct zeros is the same as subtracting off twice a 1-level sum over all zeros. This would be false if the functional equation were odd. In that case we would have to add back $g_{1}(0) g_{2}(0)$ for the extra zero at the central point, and in fact the presence or absence of this additional term is the cause of the differences in the 2-level densities of the three orthogonal groups. 


\section{The LOW LYING ZEROS OF TWO FAMILIES OF $L$-FUNCTIONS}

For the first term in (3.51), since we are summing over all zeros, we may use the explicit formula for the sum over each $j_{i}$. Let

$$
\begin{aligned}
b_{\phi \times \operatorname{sym}^{2} f}(p) & =a_{\phi \times \operatorname{sym}^{2} f}(p) \\
b_{\phi \times \operatorname{sym}^{2} f}\left(p^{2}\right) & =a_{\phi \times \operatorname{sym}^{2} f}\left(p^{2}\right)+1 .
\end{aligned}
$$

In the expansions with the explicit formula, we isolate the contribution from the part of the $\nu=2$ term which contributes $\frac{1}{2} g_{i}(0)$ for small support; this is the +1 term in $b_{\phi \times \operatorname{sym}^{2} f}\left(p^{2}\right)$. We have also removed the error terms arising from $\nu \geqslant 3$; the argument is standard (see [RS96, Rub01, HM06]). We are left with considering the weighted average of

$$
\prod_{i=1}^{2}\left[\left(\widehat{g}_{i}(0)+\frac{1}{2} g_{i}(0)\right)-2 \sum_{\nu_{i}=1}^{2} \sum_{p_{i}} \frac{b_{\phi \times \mathrm{sym}^{2} f}\left(p^{\nu_{i}}\right) \log p}{p^{\nu_{i} / 2} \log R} \widehat{g}_{i}\left(\nu_{i} \frac{\log p_{i}}{\log R}\right)\right] .
$$

There are three terms for each $i$. For small support, we have shown that the $\nu_{i}$-sums by themselves do not contribute, although we will see that there are contributions when a $\nu_{1}$-sum hits a $\nu_{2}$-sum. Thus, when a $\widehat{g_{i}}(0)+\frac{1}{2} g_{i}(0)$ hits a $\nu$-sum, there is no contribution. We have $\left[\widehat{g_{1}}(0)+\frac{1}{2} g_{1}(0)\right] \cdot\left[\widehat{g_{2}}(0)+\right.$ $\left.\frac{1}{2} g_{2}(0)\right]$, plus the weighted average of the mixed sums

$$
4 \sum_{p_{1}} \sum_{p_{2}} \frac{b_{\phi \times \mathrm{sym}^{2} f}\left(p_{1}^{\nu_{1}}\right) b_{\phi \times \mathrm{sym}^{2} f}\left(p_{2}^{\nu_{2}}\right) \log p_{1} \log p_{2}}{p_{1}^{\nu_{1} / 2} p_{2}^{\nu_{2} / 2} \log ^{2} R} \widehat{g_{1}}\left(\nu_{1} \frac{\log p_{1}}{\log R}\right) \widehat{g_{2}}\left(\nu_{2} \frac{\log p_{2}}{\log R}\right)
$$

for $\left(\nu_{1}, \nu_{2}\right) \in\{(1,1),(2,1),(2,1),(2,2)\}$. For each pair there are two cases, when $p_{1}=p_{2}$ and $p_{1} \neq p_{2}$. Since we are only interested in the 2-level density for arbitrarily small support as a means to distinguish $\mathrm{SO}$ (even) from orthogonal and $\mathrm{SO}$ (odd) symmetry, we do not record how large we may take the support.

\subsubsection{Terms $(1,1)$. We have}

$$
\frac{4}{\left|H_{k}\right|} \sum_{f \in H_{k}} \frac{\zeta(2)}{L\left(1, \operatorname{sym}^{2} f\right)} \sum_{p_{1}} \sum_{p_{2}} \frac{a_{\phi \times \mathrm{sym}^{2} f}\left(p_{1}\right) a_{\phi \times \mathrm{sym}^{2} f}\left(p_{2}\right) \log p_{1} \log p_{2}}{\sqrt{p_{1} p_{2}} \log ^{2} R} \widehat{g_{1}}\left(\frac{\log p_{1}}{\log R}\right) \widehat{g_{2}}\left(\frac{\log p_{2}}{\log R}\right) \text {. }
$$

As $a_{\phi \times \operatorname{sym}^{2} f}(p)=\lambda_{\phi}(p) \lambda_{f}\left(p^{2}\right)$, we have

$$
\frac{4}{\left|H_{k}\right|} \sum_{f \in H_{k}} \frac{\zeta(2)}{L\left(1, \operatorname{sym}^{2} f\right)} \sum_{p_{1}} \sum_{p_{2}} \lambda_{\phi}\left(p_{1}\right) \lambda_{\phi}\left(p_{2}\right) \frac{\lambda_{f}\left(p_{1}^{2}\right) \lambda_{f}\left(p_{2}^{2}\right) \log p_{1} \log p_{2}}{\sqrt{p_{1} p_{2}} \log ^{2} R}\left(\frac{\log p_{1}}{\log R}\right) \widehat{g_{2}}\left(\frac{\log p_{2}}{\log R}\right) \text {. }
$$

If $p_{1} \neq p_{2}$, when we use the Petersson formula there is no contribution for small support, since it is a non-diagonal term. Note $p_{1}=p_{2}$ is a diagonal term, giving

$$
\frac{1}{\left|H_{k}\right|} \sum_{f} \frac{\zeta(2)}{L\left(1, \operatorname{sym}^{2} f\right)} \lambda_{f}\left(p^{2}\right) \lambda_{f}\left(p^{2}\right)=1+O\left(\sqrt{p^{4}} / 2^{k}\right) .
$$

For sufficiently small support the error term is negligible, and thus the diagonal term is

$$
4 \sum_{p} \lambda_{\phi}(p) \lambda_{\phi}(p) \frac{\log ^{2} p}{p \log ^{2} R} \widehat{g_{1}}\left(\frac{\log p_{1}}{\log R}\right) \widehat{g_{2}}\left(\frac{\log p_{2}}{\log R}\right)+o(1) .
$$

We use $\lambda_{\phi}(p) \lambda_{\phi}(p)=1+\lambda_{\phi}\left(p^{2}\right)$; we saw in $\S 3.2 .3$ that the $\lambda_{\phi}\left(p^{2}\right)$ term will not contribute by the GRH for $L\left(s, \operatorname{sym}^{2} \phi\right)$. The +1 will contribute, with test function $\widehat{g_{1}} \widehat{g_{2}}$. By Lemma 2.7 , we have

$$
4 \sum_{p} \frac{\log ^{2} p}{p \log ^{2} R} \cdot \widehat{g_{1}} \widehat{g_{2}}\left(\frac{\log p}{\log R}\right)=2 \int|u| \widehat{g_{1}} \widehat{g_{2}}(u) d u+O\left(\frac{1}{\log R}\right) .
$$




\section{E. Dueñez And S. J. Miller}

3.3.2 Terms $(1,2)$ and $(2,1)$. As these terms are handled identically, we confine ourselves to $(1,2)$. We have weighted averages of

$$
4 \sum_{p_{1}} \sum_{p_{2}} \frac{a_{\phi \times \operatorname{sym}^{2} f}\left(p_{1}\right) b_{\phi \times \operatorname{sym}^{2} f}\left(p_{2}^{2}\right) \log p_{1} \log p_{2}}{p_{1}^{1 / 2} p_{2} \log ^{2} R} \widehat{g_{1}}\left(\frac{\log p_{1}}{\log R}\right) \widehat{g_{2}}\left(2 \frac{\log p_{2}}{\log R}\right) .
$$

By Lemma 3.3 and (3.52),

$$
\begin{aligned}
a_{\phi \times \operatorname{sym}^{2} f}(p) & =\lambda_{\phi}(p) \lambda_{f}\left(p^{2}\right) \\
b_{\phi \times \operatorname{sym}^{2} f}\left(p^{2}\right) & =\lambda_{\phi}\left(p^{2}\right)\left(\lambda_{f}\left(p^{4}\right)-\lambda_{f}\left(p^{2}\right)+1\right)-\left(\lambda_{f}\left(p^{4}\right)-\lambda_{f}\left(p^{2}\right)\right) .
\end{aligned}
$$

If $p_{1} \neq p_{2}$, all terms are non-diagonal, and the Petersson formula yields no contribution for small support.

If $p_{1}=p_{2}$, only two terms give diagonal terms:

$$
\lambda_{\phi}(p) \lambda_{\phi}\left(p^{2}\right) \cdot \lambda_{f}\left(p^{2}\right) \lambda_{f}\left(p^{2}\right) \text { and }-\lambda_{\phi}(p) \lambda_{f}\left(p^{2}\right) \lambda_{f}\left(p^{2}\right) .
$$

However, while the Petersson formula will give a \pm 1 for each of these diagonal terms, this is immaterial since we are dividing by $p^{3 / 2}$. Using the Kim-Sarnak bound of $p^{7 / 64}$ for Maass forms is enough to show there is no contribution, for any support, from these terms. We are dividing by $p^{3 / 2}$, and we have at most $p^{(7 \cdot 3) / 64} \log p$ in the numerator. This gives $p^{-1-11 / 64} \log p$, which yields a $O(1 / \log R)$ contribution. Arguing as in (3.47) to (3.49), one may replace the Kim-Sarnak bound with any non-trivial bound towards Ramanujan.

3.3.3 Term $(2,2)$. We now consider the $(2,2)$ term. We have a weighted average of

$$
4 \sum_{p_{1}} \sum_{p_{2}} \frac{b_{\phi \times \mathrm{sym}^{2} f}\left(p_{1}^{2}\right) b_{\phi \times \mathrm{sym}^{2} f}\left(p_{2}^{2}\right) \log p_{1} \log p_{2}}{p_{1} p_{2} \log ^{2} R} \widehat{g_{1}}\left(2 \frac{\log p_{1}}{\log R}\right) \widehat{g_{2}}\left(2 \frac{\log p_{2}}{\log R}\right),
$$

where, by Lemma 3.3 and (3.52),

$$
b_{\phi \times \operatorname{sym}^{2} f}\left(p^{2}\right)=\lambda_{\phi}\left(p^{2}\right) \cdot\left(\lambda_{f}\left(p^{4}\right)-\lambda_{f}\left(p^{2}\right)+1\right)-1 \cdot\left(\lambda_{f}\left(p^{4}\right)-\lambda_{f}\left(p^{2}\right)\right) .
$$

When $p_{1}=p_{2}$, as $\lambda_{\phi}(p)=O\left(p^{\delta}\right)$ for some $\delta \in\left[0, \frac{1}{2}\right], b_{\phi \times \mathrm{sym}^{2} f}\left(p^{2}\right)^{2} / p^{2}=O\left(p^{4 \delta-2}\right)$, and these terms will not contribute for any $\delta<\frac{1}{4}$. We do not need the full strength of the Kim-Sarnak bound; the $\frac{5}{28}$ of [BDHI92] suffices.

We are left with the case $p_{1} \neq p_{2}$. The only diagonal term will be $\lambda_{\phi}\left(p_{1}^{2}\right) \lambda_{\phi}\left(p_{2}^{2}\right) \cdot \lambda_{f}(1) \lambda_{f}(1)$. For small support, the other terms will not contribute, and by Petersson's formula we have

$$
4 \prod_{i=1}^{2} \sum_{p_{i}} \frac{\lambda_{\phi}\left(p_{i}^{2}\right) \log p_{i}}{p_{i} \log R} \widehat{g}_{i}\left(2 \frac{\log p_{i}}{\log R}\right) .
$$

As in $\S 3.2 .3$, by the GRH for $L\left(s, \operatorname{sym}^{2} \phi\right)$ each prime sum is $O(1 / \log R)$. Thus, there is no contribution from the $(2,2)$ terms.

3.3.4 Summary. We have shown

$D_{2, \mathcal{F}_{\phi \times \mathrm{sym}^{2} H_{k}}}(g)=\left[\widehat{g_{1}}(0)+\frac{1}{2} g_{1}(0)\right] \cdot\left[\widehat{g_{2}}(0)+\frac{1}{2} g_{2}(0)\right]+2 \int_{u}|u| \widehat{g_{1}} \widehat{g_{2}}(u) d u-2 \widehat{g_{1} g_{2}}(0)-g_{1}(0) g_{2}(0)$,

and (3.65) agrees only with the 2-level density for $\mathrm{SO}$ (even) (see (2.7)), completing the proof of Theorem 1.2. 


\section{The LOW LYING ZEROS OF TWO FAMILIES OF $L$-FUnCTIONS}

\section{The family $\mathcal{F}_{\phi \times H_{k}}=\left\{\phi \times f: f \in H_{k}\right\}$}

In this section we prove Theorem 1.1, namely that the symmetry type of the family $\mathcal{F}_{\phi \times H_{k}}=\{\phi \times f$ : $\left.f \in H_{k}\right\}(k \rightarrow \infty)$ agrees only with symplectic, where $\phi$ is a fixed even cuspidal Hecke-Maass form with eigenvalue $\lambda_{\phi}=\frac{1}{4}+t_{\phi}^{2}$ and $f \in H_{k}$ is a Hecke holomorphic modular form of weight $k$. These $L$-functions have associated Euler products of degree 4 and are indeed associated to automorphic representations of GL(4) [Ram00]. As in $\S 3.1$ we first derive the explicit formula and find the analytic conductors for the family. In order to compute the 1-level density we analyze the local parameters and find evidence for symplectic symmetry (for small support). Since the symplectic 1-level density is distinguishable from the other classical compact groups for arbitrarily small support, there is no need to investigate the 2-level density. As the arguments are similar to those for $\phi \times \operatorname{sym}^{2} f$, we merely sketch the calculations below. See also Appendix A for details of the determination of the gamma factors and signs of the functional equation.

\subsection{Logarithmic derivative, gamma factors, and the functional equation}

In terms of the Fourier coefficients $\left\{\lambda_{f}(n)\right\},\left\{\lambda_{\phi}(n)\right\}$ and the Maass eigenvalue $t_{\phi}\left(\frac{1}{4}+t_{\phi}^{2}\right.$ is the Laplacian eigenvalue), we have

$$
L(s, \phi \times f)=\zeta(2 s) \sum_{m} \lambda_{\phi}(m) \lambda_{f}(m) m^{-s}=\sum_{m} \lambda_{\phi \times f}(m) m^{-s},
$$

where

$$
\lambda_{\phi \times f}(m)=\sum_{m_{1}^{2} m_{2}=m} \lambda_{\phi}\left(m_{2}\right) \lambda_{f}\left(m_{2}\right) .
$$

The logarithmic derivative of $L(s, \phi \times f)$ is

$$
\frac{L^{\prime}}{L}(s, \phi \times f)=\sum_{m} \Lambda(m) a_{\phi \times f}(m) m^{-s},
$$

with

$$
a_{\phi \times f}\left(p^{\nu}\right)=\sum_{j=1}^{4} \tau_{p}(j)^{\nu} .
$$

The archimedean (gamma) factor is

$$
L_{\infty}(s, \phi \times f):=\Gamma_{\mathbb{R}}\left(s+i t_{\phi}+\frac{k-1}{2}\right) \Gamma_{\mathbb{R}}\left(s-i t_{\phi}+\frac{k-1}{2}\right) \Gamma_{\mathbb{R}}\left(s+i t_{\phi}+\frac{k+1}{2}\right) \Gamma_{\mathbb{R}}\left(s-i t_{\phi}+\frac{k+1}{2}\right),
$$

and the completed $L$-function

$$
\Lambda(s, \phi \times f):=L_{\infty}(s, \phi \times f) L(s, \phi \times f)
$$

satisfies the functional equation

$$
\Lambda(s, \phi \times f)=\Lambda(1-s, \phi \times f) .
$$

Note the functional equation is even. We define the archimedean parameters $\mu_{j}, 1 \leqslant j \leqslant 4$, as the numbers

$$
\frac{k \pm 1}{2} \pm i t_{\phi}
$$




\subsection{Explicit formula}

As in $\S 3.1 .1$, let $R>0$ be a parameter; later we take $R=k^{4}$. Assuming the GRH we may write the non-trivial zeros of $L(s, \phi \times f)$ as $\rho_{j}=\frac{1}{2}+i \gamma_{j}, j \in \mathbb{Z}-\{0\}$ (since all signs are even). Then

$$
\sum_{j} g\left(\frac{\gamma_{j}}{2 \pi} \log R\right)=\frac{A}{\log R}-2 \sum_{p} \sum_{\nu=1}^{\infty} \hat{g}\left(\frac{\nu \log p}{\log R}\right) \frac{a_{\phi \times f}\left(p^{\nu}\right) \log p}{p^{\nu / 2} \log R},
$$

where

$$
A=\int_{-\infty}^{\infty} \sum_{j=1}^{4}\left(\frac{\Gamma_{\mathbb{R}}^{\prime}}{\Gamma_{\mathbb{R}}}\left(\mu_{j}+\frac{1}{2}+\frac{2 \pi i x}{\log R}\right)+\frac{\Gamma_{\mathbb{R}}^{\prime}}{\Gamma_{\mathbb{R}}}\left(\overline{\mu_{j}}+\frac{1}{2}+\frac{2 \pi i x}{\log R}\right)\right) g(x) d x .
$$

An analogous calculation as in $\S 3.1 .2$ gives, up to lower-order terms, that the conductor is $\left(k^{2} / 4\right)^{2}$. As we only care about the logarithm of the conductor, we take $R=k^{4}$. The contribution to the 1-level density will be $\widehat{g}(0)$ plus lower-order terms.

\subsection{Relation of $a_{\phi \times f}$ to $\lambda_{\phi}$ and $\lambda_{f}$}

We consider the local parameters $\alpha_{p}^{ \pm 1}$ at any prime $p$ for $f$, as well as $\beta_{p}^{ \pm 1}$ for $\phi$. The local parameters $\tau_{p}(j)(j=1,2,3,4)$ for the automorphic representation associated to $L(s, \phi \times f)$ are the four numbers $\alpha_{p}^{ \pm 1} \beta_{p}^{ \pm 1}$. A calculation similar to but simpler than that in $\S 3.1 .3$ gives

$$
\begin{aligned}
a_{\phi \times f}(p) & =\lambda_{\phi}(p) \lambda_{f}(p) \\
a_{\phi \times f}\left(p^{\nu}\right) & =\left(\lambda_{\phi}\left(p^{\nu}\right)-\lambda_{\phi}\left(p^{\nu-2}\right)\left(\lambda_{f}\left(p^{\nu}\right)-\lambda_{f}\left(p^{\nu-2}\right)\right), \quad \nu \geqslant 2 .\right.
\end{aligned}
$$

In particular,

$$
a_{\phi \times f}\left(p^{2}\right)=\left(\lambda_{\phi}\left(p^{2}\right)-1\right)\left(\lambda_{f}\left(p^{2}\right)-1\right)
$$

4.3.1 Terms with $\nu \geqslant 3$. We show there is no contribution to the 1-level density from terms with $\nu \geqslant 3$ in (4.9). The Satake parameters are the four numbers $\alpha_{p}^{ \pm 1} \beta_{p}^{ \pm 1}$, each of which is bounded by $p^{\delta}$ with $\delta<\frac{1}{6}$ by Kim and Sarnak [Kim03]. Thus

$$
\frac{a_{\phi, f}\left(p^{\nu}\right)}{p^{\nu / 2}} \ll p^{\nu(\delta-1 / 2)},
$$

and, as $\delta<\frac{1}{6}$, summing over $p$ and $\nu \geqslant 3$ is $O(1)$. Dividing by $\log R=\log k^{4}$, we see there is no contribution.

4.3.2 Terms with $\nu=1$. As $a_{\phi \times f}=\lambda_{\phi}(p) \lambda_{f}(p)$ and $\log R=\log k^{4}$, we have

$$
-2 \sum_{p} \hat{g}\left(\frac{\log p}{\log R}\right) \frac{\lambda_{\phi}(p) \lambda_{f}(p) \log p}{\sqrt{p} \log R} .
$$

As $1=\lambda_{f}(1)$, summing over $f \in H_{k}$ yields no contribution for small support, as $\lambda_{f}(p) \lambda_{f}(1)$ is a non-diagonal term.

4.3.3 Terms with $\nu=2$. As $a_{\phi \times f}\left(p^{2}\right)=\left(\lambda_{\phi}\left(p^{2}\right)-1\right)\left(\lambda_{f}\left(p^{2}\right)-1\right)$, we have

$$
-2 \sum_{p} \hat{g}\left(\frac{2 \log p}{\log R}\right) \frac{\left(\lambda_{\phi}\left(p^{2}\right)-1\right)\left(\lambda_{f}\left(p^{2}\right)-1\right) \log p}{p \log R} .
$$

There are four types of terms:

$$
\lambda_{\phi}\left(p^{2}\right) \cdot \lambda_{f}\left(p^{2}\right) \lambda_{f}(1), \quad-\lambda_{\phi}\left(p^{2}\right), \quad-\lambda_{f}\left(p^{2}\right) \lambda_{f}(1), \quad(-1)(-1) .
$$




\section{ThE LOW LYING ZEROS OF TWO FAMILIES OF $L$-FUNCTIONS}

The first and third terms are non-diagonal, and by the Petersson formula will not contribute for small support. The second term is diagonal; however, by the GRH for $L\left(s, \operatorname{sym}^{2} \phi\right)$ (see (3.44)), this term is $O(1 / \log R)$. We are left with the fourth piece,

$$
-(-1)^{2} \cdot 2 \sum_{p} \hat{g}\left(\frac{2 \log p}{\log R}\right) \frac{\log p}{p \log R} .
$$

By Lemma 2.7, the $p$-sum is $g(0) / 4$; thus, the $\nu=2$ terms contribute, for small support, $-\frac{1}{2} g(0)$.

4.3.4 Summary. The previous subsections proved Theorem 1.1: that as $k \rightarrow \infty$, the 1-level density of $\mathcal{F}_{\phi \times H_{k}}$ agrees only with symplectic symmetry. We took two orthogonal families (when $k \equiv 2 \bmod 4$, then $H_{k}$ has $\mathrm{SO}\left(\right.$ odd) symmetry, and when $k \equiv 0 \bmod 4$, then $H_{k}$ has $\mathrm{SO}($ even) symmetry), and showed that their twists by a fixed even, full-level Maass form give a symplectic family. This should be compared with Theorem 1.2, where we twisted a symplectic family and obtained an $\mathrm{SO}$ (even) family.

Remark 4.1. The reason for the symmetry flipping can be found in (4.13). For the $\nu=2$ terms, the Maass form introduces an extra factor of -1 in the diagonal contribution. This changes the sign of the contribution from the $\nu=2$ terms, and switches us from symplectic to orthogonal symmetries (if we have orthogonal symmetries, we need to evaluate the 2-level density to determine which orthogonal symmetry as our supports are too small to distinguish between $\mathrm{SO}($ even), O, and $\mathrm{SO}($ odd)).

\section{Conclusion}

We have investigated the distribution of low-lying zeros for two families. The first is a GL(6) family, $\left\{\phi \times \operatorname{sym}^{2} f: f \in H_{k}\right\}$; here $\phi$ is a fixed Hecke-Maass cusp form and $k \rightarrow \infty$. Although this is a GL(6) family, only four of the six gamma factors depend on $k$, and the analytic conductor is $k^{4}$. Since all elements of this family have even functional equation and there is no natural complementary family with odd sign, a folklore conjecture predicted that the underlying group symmetry should be symplectic. However, the symmetry type is $\mathrm{SO}$ (even), proving that low-lying zeros are more than just a theory of signs of functional equations.

We have calculated the 1-level density for test functions $g$ such that $\operatorname{supp}(\widehat{g})$ is $\operatorname{small}(\operatorname{supp}(\widehat{g}) \subset$ $\left.\left(-\frac{5}{24}, \frac{5}{24}\right)\right)$. For such small support, only the diagonal terms in the Petersson formulas contribute. Thus, we can eliminate two of the five classical compact groups, namely symplectic and unitary. Unfortunately, since the support is significantly less than $(-1,1)$, all three orthogonal candidates are still possible; however, as all members of the family have even functional equation, we do not expect the underlying symmetry to be either $\mathrm{O}$ or $\mathrm{SO}$ (odd). Observe that the reason for the flipping of symmetry from symplectic to (some flavor of) orthogonal is that the contribution from the squares of primes (which is what is responsible in any case for the term $\pm \frac{1}{2} g(0)$ ) changes sign.

More precisely, in [ILS00] for the family $\left\{\operatorname{sym}^{2} f\right\}$, there is a contribution of $-\frac{1}{2} \widehat{g}(0)$, arising from the diagonal term +1 in $\lambda_{f}\left(p^{4}\right)-\lambda_{f}\left(p^{2}\right)+1$. In our family, this term is multiplied by the factor $\lambda_{\phi}\left(p^{2}\right)-1$ (see $(3.29)$ ), and the -1 results in a diagonal contribution of opposite sign, hence the symmetry flipping.

To discard the $\mathrm{O}$ and $\mathrm{SO}$ (odd) symmetries, we calculated the 2-level density. It is shown in [Mil02, Mil04] that for arbitrarily small support the 2-level densities of the three orthogonal groups are distinguishable. We see that our answer agrees only with $\mathrm{SO}$ (even), further supporting the claim that the symmetry group of this family is $\mathrm{SO}$ (even).

Our second example is a GL(4) family, $\left\{\phi \times f: f \in H_{k}\right\}$. Here, for the same reason as before, twisting flips the symmetry (this time from orthogonal to symplectic). In a subsequent paper, we will 


\section{E. Dueñez And S. J. Miller}

describe the interplay between twisting by a fixed GL( $n)$ form (or family) and the symmetry type (in certain cases). The arguments of this paper can be generalized to families satisfying certain natural technical conditions. It can be shown that a natural 'family constant' $c$ can be attached to a family in such a manner that $c_{\mathcal{F} \times \mathcal{G}}=c_{\mathcal{F}} \cdot c_{\mathcal{G}}$, where $c=0(1,-1)$ for unitary (symplectic, orthogonal) symmetry. Here $\mathcal{F} \times \mathcal{G}$ is the family obtained by Rankin-Selberg convolution of the $L$-functions in the families $\mathcal{F}$ and $\mathcal{G}$. These results are similar in spirit to the universality found by Rudnick and Sarnak [RS96] in the $n$-level correlations of high zeros, and will be described in further detail in [DM06]. Specifically, it again seems that the second moment of the Satake parameters determines the answer. For the GL(6) family, the main term from averaging the second moment of the Satake parameters (see (3.8)) is -1 and leads to $\mathrm{SO}$ (even) symmetry, while in the GL(4) family the main term (see (4.13)) is +1 and leads to symplectic symmetry.

\section{Appendix A. Gamma factors and signs of functional equations}

In this appendix we derive the precise forms $((3.9)$ and (4.5)) of the gamma factors for the completed $L$-functions $L\left(s, \phi \times \operatorname{sym}^{2} f\right)$ and $L(s, \phi \times f)$, as well as their functional equations ((3.12) and (4.7)). In particular, we show that both functional equations are even.

Being Hecke eigenforms of level 1, $f$ and $\phi$ can be identified with automorphic cuspidal representations $F$ and $\Phi$ of $\mathrm{GL}_{2}\left(\mathbb{A}_{\mathbb{Q}}\right)$ with trivial central character [Gel75]. The latter are isomorphic to restricted tensor products

$$
F \cong \prod_{v}^{\prime} F_{v}, \quad \Phi \cong \prod_{v}^{\prime} \Phi_{v}
$$

of representations of $\mathrm{GL}_{2}\left(\mathbb{Q}_{v}\right)$ for each place $v$ of $\mathbb{Q}$ (here $v=p$ for $p$ prime, or $v=\infty$, in which case $\mathbb{Q}_{\infty}=\mathbb{R}$ ). In the case at hand, as every (finite) prime $p$ is unramified, the corresponding principal series representations of $\mathrm{GL}_{2}\left(\mathbb{Q}_{p}\right)$ have associated $\mathrm{SL}_{2}(\mathbb{C})$-conjugacy classes ${ }^{1}$

$$
F_{p} \leftrightarrow\left(\begin{array}{cc}
\alpha_{p} & 0 \\
0 & \alpha_{p}^{-1}
\end{array}\right)^{\natural}, \quad \Phi_{p} \leftrightarrow\left(\begin{array}{cc}
\beta_{p} & 0 \\
0 & \beta_{p}^{-1}
\end{array}\right)^{\natural} .
$$

Denote by $M\left(\alpha_{p}\right), M\left(\beta_{p}\right)$ the matrices in (A.2).

Taking the symmetric square of the standard representation of $\mathrm{GL}_{2}(\mathbb{R})$, one obtains the conjugacy class $\operatorname{sym}^{2} M\left(\alpha_{p}\right)^{\natural}=\operatorname{diag}\left(\alpha_{p}^{2}, 1, \alpha_{p}^{-2}\right)^{\natural}$, whence the Satake parameters of $\operatorname{sym}^{2} f$. Similarly, the conjugacy classes of $M\left(\alpha_{p}\right) \otimes M\left(\beta_{p}\right)$ in $\mathrm{GL}_{4}(\mathbb{R})$ and $\operatorname{sym}^{2} M\left(\alpha_{p}\right) \otimes M\left(\beta_{p}\right)$ in $\mathrm{GL}_{6}(\mathbb{R})$ define the Satake parameters of $\phi \times f$ and $\phi \times \operatorname{sym}^{2} f$. The local $L$-factors are defined in terms of these Satake parameters in the usual manner, and the product of all local factors defines the (incomplete) $L$-functions (3.5) and (4.1).

The representations $F_{\infty}$ and $\Phi_{\infty}$ are the discrete series representation of weight $k$ and the representation $I\left(|\cdot|^{i t},|\cdot|^{-i t}\right.$ ) of $\mathrm{GL}_{2}(\mathbb{R})$, respectively (recall that $\frac{1}{4}+t^{2}$ is the Laplacian eigenvalue of $\phi) .{ }^{2}$ Selberg proved that $t$ is real for Maass forms of level 1 (and conjectured that $t$ is still real for forms of any weight). The first published proof is due to Roelcke [Roe56]; see also [Iwa95].

Proofs of (3.9) and (4.5) involve parametrizing the representations $F_{\infty}$ and $\Phi_{\infty}$, through the Langlands correspondence, by semisimple representations of the Weil group $W_{\mathbb{R}}$ (see [Kna94, CM04]). We number the discrete series as in [Kna94] (see the note at the top of p. 1588 of [CM04]), so replacing $k$ by $\ell+1$ in the following would make our notation agree with that of [CM04].

\footnotetext{
${ }^{1} A^{\natural}$ denotes the conjugacy class of $A$.

${ }^{2} I\left(|\cdot|^{i t},|\cdot|^{-i t}\right)$ is the unitary induction from the group $Q$ of upper-triangular matrices to $\mathrm{GL}_{2}(\mathbb{R})$ of the representation $\left(\begin{array}{c}a * \\ d\end{array}\right) \mapsto|a|^{i t}|d|^{-i t}$
} 
Then (cf. [Kna94, (3.2) and (3.3)])

$$
\begin{aligned}
& F_{\infty} \leftrightarrow \rho_{(k-1,0)}, \\
& \Phi_{\infty} \leftrightarrow \rho_{(+, i t)} \oplus \rho_{(+,-i t)} .
\end{aligned}
$$

We have denoted by $\rho_{(a, b)}$ the semisimple representation of $W_{\mathbb{R}}$ with parameters $(a, b)$. The known cases of functoriality [GJ78, Ram00, KS02] imply the existence of automorphic (cuspidal) representations $\operatorname{sym}^{2} F, \Phi \times \operatorname{sym}^{2} F$, and $\Phi \times F$ such that, by the archimedean Langlands correspondence,

$$
\begin{aligned}
\left(\operatorname{sym}^{2} F\right)_{\infty} & \leftrightarrow \operatorname{sym}^{2} \rho_{(k-1,0)} \\
\left(\Phi \times \operatorname{sym}^{2} F\right)_{\infty} & \leftrightarrow\left(\rho_{(+, i t)} \oplus \rho_{(+,-i t)}\right) \otimes \operatorname{sym}^{2} \rho_{(k-1,0)} \\
(\Phi \times F)_{\infty} & \leftrightarrow\left(\rho_{(+, i t)} \oplus \rho_{(+,-i t)}\right) \otimes \rho_{(k-1,0)} .
\end{aligned}
$$

By [CM04, Proposition 3.1], ${ }^{3}$

$$
\operatorname{sym}^{2} \rho_{(k-1,0)} \cong \rho_{(-, 0)} \oplus \rho_{(2 k, 0)} .
$$

Moreover, it is easily checked that

$$
\begin{aligned}
& \rho_{(+, \pm i t)} \otimes \rho_{(-, 0)} \cong \rho_{(-, \pm i t)} \\
& \rho_{(+, \pm i t)} \otimes \rho_{(\ell, 0)} \cong \rho_{(\ell, \pm i t)} .
\end{aligned}
$$

Hence

$$
\begin{aligned}
\left(\Phi \times \operatorname{sym}^{2} F\right)_{\infty} & \leftrightarrow \rho_{(-, i t)} \oplus \rho_{(-,-i t)} \oplus \rho_{(2 k-2, i t)} \oplus \rho_{(2 k-2,-i t)} \\
(\Phi \times F)_{\infty} & \leftrightarrow \rho_{(k-1, i t)} \oplus \rho_{(k-1,-i t)} .
\end{aligned}
$$

The archimedean (gamma) factors can be found using these decompositions and the local Langlands correspondence. In terms of irreducible semisimple representations of $W_{\mathbb{R}}$, we have

$$
L(s, \rho)= \begin{cases}\Gamma_{\mathbb{R}}(s \pm i t) & \rho=\rho_{(+, \pm i t)}, \\ \Gamma_{\mathbb{R}}(s \pm i t+1) & \rho=\rho_{(-, \pm i t)} \\ \Gamma_{\mathbb{R}}\left(s \pm i t+\frac{\ell}{2}\right) \Gamma_{\mathbb{R}}\left(s \pm i t+\frac{\ell}{2}+1\right) & \rho=\rho_{(\ell, \pm i t)} .\end{cases}
$$

These local factors are multiplicative under direct sums of representations of $W_{\mathbb{R}}$, so definitions (3.9) and (4.5) are consistent with (A.11) and (A.12) via (A.13).

Since all automorphic representations under discussion are self-contragredient, the functional equations relate each $L$-function to itself as $s \mapsto 1-s$. In general, the root number $\varepsilon(s, \pi)$ associated to an automorphic cuspidal representation $\pi$ is a product

$$
\varepsilon(s, \pi)=\prod_{v} \varepsilon\left(s, \pi_{v}\right)
$$

of local root numbers. ${ }^{4}$ For self-contragredient representations $\pi=\tilde{\pi}$ the $\varepsilon$-factor agrees with the sign of the functional equation (up to a factor $Q^{s}$ which is not present in the level-1 case that concerns us). Moreover, local root numbers can be computed via the local Langlands correspondence as root numbers associated to Weil group representations. In addition, $\varepsilon\left(s, \pi_{p}\right)=1$ at any prime $p$ such

\footnotetext{
${ }^{3}$ Note that the weight $k$ of our $f$ is always even. Thus, the appearance of $(-, 0)$ in $($ A.8) is due to the fact that $(-1)^{k-1}=-1$.

${ }^{4}$ We have omitted the dependence of the local root numbers on the choice of additive character $\psi$ of $\mathbb{A}_{\mathbb{Q}}$.
} 


\section{E. Dueñez And S. J. Miller}

that $\pi_{p}$ is unramified. ${ }^{5}$ The local root number of an irreducible representation $\rho$ of $W_{\mathbb{R}}$ is

$$
\varepsilon(s, \rho)= \begin{cases}1 & \rho=\rho_{(+, \pm i t)}, \\ i & \rho=\rho_{(-, \pm i t)}, \\ i^{\ell+1} & \rho=\rho_{(\ell, \pm i t)} .\end{cases}
$$

From (A.14) and (A.11), (A.12) we obtain

$$
\varepsilon\left(\left(\Phi \times \operatorname{sym}^{2} F\right)_{\infty}\right)=i \cdot i \cdot i^{2 k-1} \cdot i^{2 k-1}=+1,
$$

and, since $k$ is even,

$$
\varepsilon\left((\Phi \times F)_{\infty}\right)=i^{k} \cdot i^{k}=+1 .
$$

Since all (finite) primes $p$ are unramified for $\Phi \times F$ and $\Phi \times \operatorname{sym}^{2} F$, we conclude that

$$
\varepsilon\left(s, \Phi \times \operatorname{sym}^{2} F\right)=\varepsilon(s, \Phi \times F)=+1,
$$

so the global functional equations have even sign, proving (3.12) and (4.7).

\section{REFERENCES}

Bum84 D. Bump, Automorphic forms on $\mathrm{GL}(3, \mathbb{R})$, Lecture Notes in Mathematics, vol. 1083 (Springer, Berlin, 1984).

Bum89 D. Bump, The Rankin-Selberg method: a survey, in Number theory, trace formulas and discrete groups, Oslo, 1987 (Academic Press, Boston, MA, 1989), 49-109.

BDHI92 D. Bump, W. Duke, J. Hoffstein and H. Iwaniec, An estimate for the Hecke eigenvalues of Maass forms, Internat. Math. Res. Notices 4 (1992), 75-81.

CM04 J. Cogdell and P. Michel, On the complex moments of symmetric power L-functions at $s=1$, Internat. Math. Res. Notices 31 (2004), 1561-1617.

CFKRS05 B. Conrey, D. Farmer, J. P. Keating, M. Rubinstein and N. Snaith, Integral moments of L-functions, Proc. London Math. Soc. (3) 91 (2005), 33-104.

DM06 E. Dueñez and S. J. Miller, The effect of convolving families of L-functions on the underlying group symmetries, Preprint (2006).

FI03 E. Fouvry and H. Iwaniec, Low-lying zeros of dihedral L-functions, Duke Math. J. 116 (2003), 189-217.

Gel75 S. Gelbart, Automorphic forms on adèle groups, Annals of Mathematics Studies, vol. 83 (Princeton University Press, Princeton, NJ, 1975).

GJ78 S. Gelbart and H. Jacquet, A relation between automorphic representations of GL(2) and GL(3), Ann. Sci. École Norm. Sup. (4) 11 (1978), 471-542.

GR65 I. Gradshteyn and I. Ryzhik, Tables of integrals, series, and products (Academic Press, New York, 1965).

Gul05 A. Güloğlu, Low lying zeros of symmetric power L-functions, Internat. Math. Res. Notices 9 (2005), 517-550.

Hej94 D. Hejhal, On the triple correlation of zeros of the zeta function, Internat. Math. Res. Notices 7 (1994), 294-302.

HM06 C. Hughes and S. J. Miller, Low-lying zeros of L-functions with orthogonal symmetry, Duke Math. J., to appear, http://arxiv.org/pdf/math.NT/0507450.

HR03 C. Hughes and Z. Rudnick, Linear statistics of low-lying zeros of L-functions, Q. J. Math. 54 (2003), 309-333.

Iwa95 H. Iwaniec, Introduction to the spectral theory of automorphic forms (Biblioteca de la Revista Matemática Iberoamericana, Madrid, 1995).

Iwa97 H. Iwaniec, Topics in classical automorphic forms, Graduate Studies in Mathematics, vol. 17 (American Mathematical Society, Providence, RI, 1997).

\footnotetext{
${ }^{5}$ We assume that $\psi_{p}$ is unramified at all primes $p$.
} 


\section{The LOW LYING ZEROS OF TWO FAMILIES OF $L$-FUNCTIONS}

ILS00 H. Iwaniec, W. Luo and P. Sarnak, Low lying zeros of families of L-functions, Publ. Math. Inst. Hautes Études Sci. 91 (2000), 55-131.

KS99a N. Katz and P. Sarnak, Random matrices, Frobenius eigenvalues and monodromy, American Mathematical Society Colloquium Publications, vol. 45 (American Mathematical Society, Providence, RI, 1999).

KS99b N. Katz and P. Sarnak, Zeros of zeta functions and symmetries, Bull. Amer. Math. Soc. (NS) 36 (1999), 1-26.

KS03 J. P. Keating and N. C. Snaith, Random matrices and L-functions, in Random matrix theory, J. Phys. A 36 (2003), 2859-2881.

Kim03 H. Kim, Functoriality for the exterior square of $\mathrm{GL}_{2}$ and the symmetric fourth of $\mathrm{GL}_{2}$, J. Amer. Math. Soc. 16 (2003), 139-183.

KS02 H. Kim and F. Shahidi, Functorial products for $\mathrm{GL}_{2} \times \mathrm{GL}_{3}$ and the symmetric cube for $\mathrm{GL}_{2}$, Ann. of Math. (2) 155 (2002), 837-893.

Kna94 A. W. Knapp, Local Langlands correspondence: the archimedean case, Proceedings of Symposia in Pure Mathematics, vol. 55, part 2 (American Mathematical Society, Providence, RI, 1994), $393-410$.

LS03 W. Luo and P. Sarnak, Mass equidistribution for Hecke eigenforms, Comm. Pure Appl. Math. 56 (2003), 874-891.

Mil02 S. J. Miller, 1- and 2-level densities for families of elliptic curves: evidence for the underlying group symmetries, $\mathrm{PhD}$ thesis, Princeton University (2002),

http://www.math.princeton.edu/ sjmiller/thesis/thesis.html.

Mil04 S. J. Miller, 1- and 2-level densities for families of elliptic curves: evidence for the underlying group symmetries, Compositio Math. 104 (2004), 952-992.

Mon73 H. Montgomery, The pair correlation of zeros of the zeta function, in Analytic number theory, Proceedings of Symposia in Pure Mathematics, vol. 24 (American Mathematical Society, Providence, RI, 1994), 181-193.

Od101 A. Odlyzko, The $10^{22}$-nd zero of the Riemann zeta function, in Dynamical, spectral and arithmetic zeta-functions, eds M. van Frankenhuysen and M. L. Lapidus, Contemporary Mathematics, vol. 290 (American Mathematical Society, Providence, RI, 2001), http://www.research.att.com/ amo/doc/zeta.html.

Ram00 D. Ramakrishnan, Modularity of the Rankin-Selberg L-series, and multiplicity one for $\mathrm{SL}(2)$, Ann. of Math. (2) 152 (2000), 45-111.

Roe56 W. Roelcke, Über die Wellengleichung bei Grenzkreisgruppen erster Art., S.-B. Heidelberger Akad. Wiss. Math. Nat. Kl. 4 (1956), 159-267.

Roy01 E. Royer, Petits zéros de fonctions L de formes modulaires, Acta Arith. 99 (2001), 147-172.

Rub98 M. Rubinstein, Evidence for a spectral interpretation of the zeros of L-functions, PhD thesis, Princeton University (1998), http://www.math.uwaterloo.ca/ mrubinst/thesis/thesis.html.

Rub01 M. Rubinstein, Low-lying zeros of L-functions and random matrix theory, Duke Math. J. 109 (2001), 147-181.

RS96 Z. Rudnick and P. Sarnak, Zeros of principal L-functions and random matrix theory, Duke Math. J. 81 (1996), 269-322.

Sil98 J. Silverman, The average rank of an algebraic family of elliptic curves, J. reine angew. Math. 504 (1998), 227-236.

You06 M. Young, Low lying zeros of families of elliptic curves, J. Amer. Math. Soc. 19 (2006), 205-250.

Eduardo Dueñez eduenez@math.utsa.edu

Department of Mathematics, The University of Texas at San Antonio, San Antonio, TX 78249, USA

Steven J. Miller sjmiller@math.brown.edu

Department of Mathematics, Brown University, 151 Thayer Street, Providence, RI 02912, USA 